\title{
Simmetrie e intertestualità teoretiche. Henry Bauchau fra dinamiche di elaborazione poetica e stilemi teologici
}

\section{Michele Mastroianni}

\author{
(2) OpenEdition \\ Journals \\ Edizione digitale \\ URL: http://journals.openedition.org/studifrancesi/8797 \\ DOI: 10.4000/studifrancesi.8797 \\ ISSN: 2421-5856 \\ Editore \\ Rosenberg \& Sellier

\section{Edizione cartacea} \\ Data di pubblicazione: 1 octobre 2008 \\ Paginazione: 336-359 \\ ISSN: 0039-2944

\section{Notizia bibliografica digitale} \\ Michele Mastroianni, «Simmetrie e intertestualità teoretiche. Henry Bauchau fra dinamiche di \\ elaborazione poetica e stilemi teologici», Studi Francesi [Online], 155 (LII | II) | 2008, online dal 30 \\ novembre 2015, consultato il 13 janvier 2021. URL: http://journals.openedition.org/studifrancesi/8797 \\ ; DOI: https://doi.org/10.4000/studifrancesi.8797
}

\section{(c)}

Studi Francesi è distribuita con Licenza Creative Commons Attribuzione - Non commerciale - Non opere derivate 4.0 Internazionale. 


\title{
Simmetrie e intertestualità teoretiche. Henry Bauchau fra dinamiche di elaborazione poetica e stilemi teologici
}

\begin{abstract}
Dalla lettura dei vari Journaux e delle pagine critiche di Henry Bauchau ${ }^{1}$ si delinea un sovrapporsi continuo di due diversi piani semantici. L'attenzione, da un lato, verso problemi di poetica - e di tutto ciò che la 'poetica' comporta a livello di linguaggio -, dall'altro un costante slittamento verso l'ambito della spiritualità, con l'utilizzazione di un lessico (e in alcuni casi di un immaginario) di derivazione biblica e teologica: di una teologia à la page negli anni sessanta; quella stessa che Bauchau avrebbe potuto conoscere, la cui frequentazione eventuale, però, non necessariamente comporta un'assunzione ideologica. Tale sovrapposizione di livelli è evidenziata $\mathrm{da}$ affermazioni che possono nascere come annotazioni di critica letteraria, ma che possono poi farsi materia costitutiva di un tessuto linguistico-letterario (o perlomeno di riferimenti) di ascendenza semantica anche teologica. Tentare di ricostruire il comporsi - per intersezioni e intertestualità - dei due suddetti livelli, può pertanto essere utile ad introdurci in un complesso laboratorio di scrittura: sicuramente all'interno di una elaborazione diversamente stratificata, debitrice di forme ermeneutiche eterogenee, saldamente intrecciate.
\end{abstract}

\section{Psicanalisi e scrittura poetica}

Con la psicanalisi Bauchau ${ }^{2}$ compie un passo decisivo verso la scrittura. Così egli annota in una pagina del Journal d'Antigone:

(1) Le opere di Henry Bauchau, cui si fa riferimento, sono indicate con le seguenti sigle: SO: $\mathrm{La}$ Sourde Oreille ou le rêve de Freud, Lausanne, Éditions de L'Aire, 1981; EC: L'Écriture et la circonstance, Louvain-la-Neuve, Chaire de poétique de l'Université Catholique de Louvain, 1988; JJ: Jour après jour. Journal d' «Edipe sur la route» (19831989), Bruxelles, Les Éperonniers, 1992; JA: Journal d'«Antigone» (1989-1997), Arles, Actes Sud, 1999; EE: L'Écriture à l'écoute, Arles, Actes Sud, 2000; PBG: Passage de la Bonne-Graine. Journal (1997-2001), Arles, Acte Sud, 2002; GM: La Grande Muraille. Journal de la «Déchirure» (1960-1965), Arles, Actes Sud, 2005.

(2) Per una bibliografia essenziale sull'autore cfr. almeno: J. Tordeur, Henry Bauchau: une mémoire de l'inconscient, «La Revue Générale», 2 (février 1986), pp. 19-27; Henry Bauchau. Un écrivain, une cuvre, «Atti del Centro Studi sulla letteratura belga di lingua francese (Terzo Seminario Internazionale, Noci, 8-10 novembre 1991)», a cura di A. SonCINI FratTA, Bologna, CLUEB («Belœil»), 1993 (cfr. di questo studio l'ampia bibliografia su Bauchau: pp. 371-382); M. WattheE-DelmotTe, Henry Bauchau. Un livre: "LEdipe sur la route». Une oewvre, Bruxelles, Labor, 1994; ID., Parcours d'Henry Bauchau, Paris, L'Harmattan, 2001 (cfr. di questo studio l'ampia bibliografia: pp. 163-204); ID. (dir.), Bauchau avant Bauchau. En amont de l'ouvre littéraire, Louvain, Academia-Bruylant, 2002; M. Watthee-DelmotTe - J. Poirer, Pierre Jean Jouve et Henry Bauchau: les voix de l'altérité, Dijon, Éditions Universitaires de Dijon («Écritures»), 2006; CH. Elefante, Mito e luoghi dell'immaginario nell'opera di Henry Bauchau, Tesi di Dottorato, Bologna, Università degli Studi di Bologna, 1995; ID., La scrittura del sogno della scrittura (a proposito di Henry Bauchau), in Jeux de langue. Jeux d'écritures, Bologna, CLUEB («Belœil»), 1995, pp. 129-151; H. BAUCHAU, Dal naturale della mano, a cura di A. MARCHETTI, Bologna, Book Editore, 1999, in particolare pp. 8-15; A. Marchetti (dir.), Prédilictions. Incursions en Belgique, Rimini, Panozzo Editore, 2002, pp. 133 183; i numeri monografici Henry Bauchau. Voix et 
[...] je me suis accroché à l'écriture, à l'aide de l'analyse pour survivre. Pour découvrir peu à peu un sens obscur au chemin que je suis, à celui que j'ai suivi .

Se la scrittura è per Bauchau riconducibile a un tentativo ultimo di sopravvivenza («pour survivre»), la psicanalisi rappresenterebbe il mezzo attraverso cui concretizzare tale percorso verso la salvezza e la creazione poetica. Esplicita affermazione, questa, che rivisita quanto egli aveva scritto nel marzo del 1960:

L'analyse fait ressurgir le passé, le transforme et lui donne une physionomie et une signification nouvelles. Le passé continue, à partir de là, à vivre et à évoluer. Le temps passé est aussi un temps qui revient "modifié", un temps qui arrive. Il s'agit de l'histoire non pas d'une guérison - je n'en suis pas là, je n'y serai peut-être jamais - mais d'une marche vers la guérison jusqu'au moment où la force emprisonnée commence à se libérer et vole au secours du "moi" défaillant ${ }^{4}$.

Non solo. Attraverso l'esplorazione dell'inconscio l'autore ricupera il senso della vita presente e passata «pour découvrir peu à peu [le] chemin que je suis... celui que j'ai suivi» e finisce con associare la sua esistenza all'idea-immagine della route, ideaimmagine di rilievo, è indubbio, all'interno della sua scrittura. Ora, se dall'analyse Bauchau passa all'écriture, da questa alla sua oggettivazione in opera letteraria noi siamo introdotti all'interno di un secondo processo di identificazione, questa volta stabilito attraverso un parallelismo fra cuvre e route, là dove l'opera diventerebbe allegoria della strada: del cammino cioè lungo cui passa ed è passata l'esperienza esistenziale, che attraverso il filtro dell'elaborazione letteraria è riscoperta in una veste nuova e diversa:

Mon œuvre est avant tout ma route, je la propose aux autres, mais c'est d'abord à moi qu'elle est utile, qu'elle a été nécessaire. Elle a été, après le temps des passions turbulentes, le moyen de me découvrir, de sortir du petit espace du moi, pour en apercevoir, très confusément ancore, un plus vaste 5

Del resto, già nel gennaio del 1960, conversando con Blanche Jouve, Bauchau estrinsecava il senso di frustrazione, maturato nel tempo, per essersi deciso a prati-

vocation de l'écriture, «Francofonia», 42 (primavera 2002) e Henry Bauchau en Suisse, «Écriture», 61 (primavera 2003); AA. Vv., Les Constellations Impérieuses d'Henry Bauchau, «Actes publiés sous la direction de M. QuagheBEur (Colloque de Cerisyla-Salle, 21-31 juillet 2001)», Bruxelles, Archives et Musées de la Littérature («Collection Archives du futur»), 2003; AA. Vv., Henry Bauchau, une poétique de l'espérance «Actes du Colloque International de Metz publiés sous la direction de P. Halen - R. Michel - M. Michel (6-8 novembre 2002)», Berne, Peter Lang, (n. 7 «Recherches en littérature et spiritualité»), 2004; G. Henrot Sostero, Henry Bauchau poète. Le vertige du seuil, Genève, Droz, 2003 (cfr. di questo studio l'ampia bibliografia: pp. 169-179); O. Ammour-MaYeur, Les Imaginaires métisses. Passages d'Extrême-Orient et d'Occident chez Henry Bauchau et Marguerite Duras, Paris, L'Harmattan, 2004; ID., Henry Bauchau, une écriture en résistance, Paris, L'Harmattan, 2006; «Nu(e)», 35 (mars 2007). È questo un numero interamente consacrato a Henry Bauchau. Oltre all'interesse per un certo materiale epistolare inedito in esso pubblicato, tale numero merita sicuramente attenzione poiché contiene alcuni saggi critici sulla scrittura dell'autore (a questo proposito cfr. pp. 185-274). Infine, anche se non implica direttamente uno studio su Bauchau, segnaliamo l'opera di J. BuRgos, Pour une poétique de l'imaginaire, Paris, Eitions du Seuil, 1982. Si tratta infatti di un saggio che indaga i problemi concernenti l'elaborazione poetica, in relazione alle problematiche teoriche che sottendono la fitta complessità subliminale ed elaborativa del discorso estetico e poetico in generale. Di quelle stesse problematiche che, costantemente, emergono negli scritti di Bauchau. Quando il presente articolo era in bozze è stato pubblicato un importante studio su Bauchau a cui ora rimandiamo: $\mathrm{R}$. LEFORT, L'originel dans l'ouvre d'Henry Bauchau, Paris, Champion, 2007.

(3) JA, p. 348, alla data 17 agosto [1994].

(4) GM, p. 36, alla data 3 marzo [1960].

(5) $J A$, p. 348, alla data 17 agosto [1994]. 
care la scrittura così tardivamente. Scrittura la cui realizzazione finale ed oggettiva nell'opera letteraria si configura immediatamente come momento di coincidenza, sì, con la maturazione poetica, ma di una maturazione probabilmente non disgiunta da una necessaria evoluzione esistenziale ad essa certamente connessa:

Je lui [scil. Blanche Jouve] parle de l'impatience de sentir le temps couler et d'avoir fait si peu de choses parce que j'ai commencé trop tard. Elle me dit: «L'œuvre chez vous est le fruit d'une lente maturation plus que de toute autre chose. Il ne faut pas vous impatienter, ni forcer» ${ }^{6}$.

Di questa scrittura, dunque, la prima tappa indotta dall'esercizio psicanalitico è segnata da un tentativo di approccio alla poesia ${ }^{7}$, praticata per brevi sprazzi interrotti e frammenti di esercizi marginali o isolati. Le cause di questa frammentarietà nella scrittura poetica sono molteplici, anzitutto di stampo generazionale:

Il est certain qu'il y a eu dans la rupture de vie subie par mon père lorsqu'il n'a pu entrer à l'École militaire un acte déterminant de sa vie. Ce fut une rupture que Jean [si tratta del fratello maggiore] et moi avons tenté de tranformer en rupture plus complète. Comme mon père s'est privé de l'armée, sa véritable vocation, je me suis longtemps privé d'écrire ${ }^{8}$,

ma anche di natura esistenziale:

[...] ce sont de justes obstacles intérieurs qui m’ont empêché d'écrire jusqu'à trente-six ans. Si j'avais pu pousser plus loin mes tentatives précédentes, je n'aurais rien écrit de valable?.

La psicanalisi è per Bauchau motore generativo, causa scatenante il jaillissement della scrittura. Ma se l'inconfutabilità di questo dato - inconfutabile nella oggettività della relazione che esso richiama tra psicanalisi e scrittura appunto - non si può negare, altrettanto evidente all'interno del nesso psycanalyse / écriture è l'appello all'infanzia proprio per il tramite della terapia sull'inconscio. Infatti il poema, alla sua origine, è il non detto, l'indicibile messo in causa dall'infanzia e che dall'infanzia prende forma - attraverso i canali delle sedute psicanalitiche posteriori - per

(6) GM, p. 18, alla data 27 gennaio [1960].

(7) Bauchau, in un saggio successivo al primo periodo in cui dispiega energie per la realizzazione di alcune composizioni poetiche di cui forse presagisce la pubblicazione, offre una spiegazione convincente circa la scelta di questo genere letterario: «C'est dans le monde disloqué où l'angoisse m'avait fait entrer, dans ce labyrinthe de l'analyse qui n'avait pas de fil d'Ariane pour revenir en arrière que je me suis mis à écrire, ou peut-être à apprendre à écrire des vers $[\ldots]$. Pourquoi des vers, pourquoi la poésie plutôt que la prose? Je me posais incessamment cette question. Pour y répondre il eût fallu savoir ce qu'était la poésie, ce que signifiait en moi le vers. Mais j'étais bien incapable de les penser, je pouvais seulement les vivre, et les éprouver comme une exigence intérieure. Il me semble aujourd'hui que je me suis tourné alors vers la poésie parce qu'elle vient de plus loin, d'une étendue plus profonde de l'histoire et de la préhistoire humaine. Parce qu'elle me reliait à des couches plus originelles de ma géologie personnelle» (EC, pp. 5-6). Non è un caso infatti che la prima raccolta poetica dell'autore, ultimata nel 1957 e pubblicata un anno dopo, porti il titolo di Géologie (per alcune informazioni sulla nascita di questa opera cfr. EC, pp. 13-14 e p. 20), proprio sulla scorta di un'idea principe secondo cui la poesia, nata dal profondo, dal profondo scava nella storia del poeta e lo riconduce a una nuova géologie personnelle, a un'autre forme de vie. Sempre sulla scelta della poesia come genere Bauchau scrive: «c'est seulement dans le poème, ai-je pensé en écrivant, qu'on travaille réellement les mots au contact de la matière intérieure, encore brûlante» (JA, p. 108, alla data 14 agosto [1991]). Per un discorso più articolato concernente le dinamiche sottese alla elaborazione della scrittura in Bauchau in relazione alla sua attività di poeta, rimandiamo al saggio di recente pubblicazione di Geneviève Henrot Sostero (op. cit.).

(8) GM, p. 26, alla data 1 febbraio [1960].

(9) JJ, p. 27, alla data 31 luglio [1984]. 
le vie della vita, innescando un processo di dipendenza che è vincolo d'amore per la scrittura ${ }^{10}$.

Tuttavia, fin dagli esordi, le motivazioni furono probabilmente connesse anche a problemi editoriali. Non è certamente un caso che la tarda fama, ma poi anche il sospirato successo di scrittore siano legati alla produzione narrativa di Bauchau e non a quella di poeta. Basti in tal senso ricordare ciò che Bauchau dice nel luglio del 1986, in un passo ove imputa allo scarso interesse che muove il lettore moderno verso la poesia parte della ragione del suo insuccesso:

Un mot d'Alain Bosquet me touche. Il me dit regretter de n'avoir pu faire plus pour mon œuvre, qu'il admire. Dans cette traversée du silence qu'à été la publication de la plupart de mes livres de poèmes, son attention soutenue n'a céssé pourtant de m'accompagner. C'est un signe du temps que le silence grandisse autour de la poésie. De la part sans doute la plus glorieuse de nos littératures et certainement de la littérature française de ce siècle ${ }^{11}$.

Nonostante ciò, l'indifferenza e la «dérision» che circondano il mondo della poesia non incrinano il suo rapporto con essa. Alcuni anni più tardi, infatti, in Jour après jour Bauchau ritornerà sull'argomento, puntualizzando e ridefinendo la sua posizione vis-à-vis della poesia stessa:

J'ai consacré cette semaine au nouveau poème de forme brève dont l'inspiration m'est venue subitement. La forme m'a été dictée à contrecœur, à cause de sa difficulté extrême, par celle de L'inattendu d'avril. Je comptais faire un poème court et c'est un poème de trente-huit vers, Corps mystique de mai, qui s'est imposé à moi. Je suis allé de nouveau à la limite de mes forces. J'en ai ressenti de la joie, sur le fond de dérision qu'il y a à notre époque à écrire des poèmes et pis, des poèmes rimés. Mais c'était un travail intérieurement commandé, auquel je ne pouvais qu'obéir ${ }^{12}$.

Bauchau stesso d'altronde ricorda come i suoi primi tentativi poetici risalgano agli anni precedenti la Seconda guerra mondiale, quando per la scarsa consuetudine con l'analisi psicanalitica non riusciva a valicare le surfaces di una elaborazione poetica lontana dall'originalità sperata:

[...] j’avais écrit, dans les années qui ont précédé la seconde guerre mondiale, quelques poèmes et des articles mais qui ne s'inscrivaient pas dans une forme originelle ni un véritable son de voix. Je n'exprimais que des surfaces, je n'entendais ni l'espérance de mon passé ni, comme me l'a dit un jour un rêve ${ }^{13}$, la mémoire de mon futur. C'est seulement lorsque l'obscurité ${ }^{14}$ est

(10) Cfr. EC, pp. 1-5: «Le souvenir de la scène dominante de mon enfance ne m'est revenu qu'à la fin de ma première analyse [...] l'enfance renvoie à l'inoubliant ce qui a eu lieu d'indicible et que toute une vie va devoir tenter de dire. C'est là que commence la dépendance amoureuse du poème [...]. L'enfance, l'existence, l'écriture ont été scandées par un incessant "jamais je n'arriverai jusque-là" auquel il fallait parvenir, auquel on parvenait peutêtre puisque la vie semblait continuer et auquel pourtant on ne parvenait pas. C'était, au-delà du désir, la nécessité de dire l'indicible, l'indicible du vécu, avec les moyens de langage élaborés pour le dicible et la vie courante. C'était l'espérance de cette impossible écriture de l'amour qui donnait le courage d'aller d'une scéance à l'autre, puis d'une année à l'autre, d'un demi-succès à un demi échec et pour tout dire de résistance en résistance».
(11) JJ, p. 150, alla data 24 luglio [1986].

(12) JA, p. 404, alla data 14 maggio [1995].

(13) Il sogno come fonte creativa è centrale nel processo di scrittura. In un testo chiave, il poème del 1981 La Sourde Oreille ou le rêve de Freud, Bauchau ricostruisce il processo che lo porta alla scrittura: partendo dall'evocazione di Freud - evocazione fatta mediante l'immagine del sogno - egli giunge al rapporto con la Sibylle (= Blanche Jouve), con l'analista cioè che attraverso l'interpretazione dei suoi sogni lo induce alla 'perseveranza' («elle te dit de persévérer», SO, p. 46) nella scrittura.

(14) Sul tema dell'oscurità come materia della scrittura cfr. almeno $J A$, pp. 152-153, alla data 23 aprile [1992]: «Péguy: "Avant les Béatitudes, il faut avoir été charpentier pendant quinze ans et avoir travaillé la matière". Le vitrail travaille la lumière, l'écriture l'obscurité intérieure». L'immagine del- 
devenue plus profonde, au cours de l'analyse et du temps des séances, que l'écriture a pu très lentement réapparaître ${ }^{15}$.

Anche altrove, d'altronde, Bauchau insiste sulla sostanziale immaturità creativa che contraddistingue gli scritti precedenti il romanzo La Déchirure (del 1966) ${ }^{16}$, pur sottolineando, in passi diversi, come già verso il $1948-49^{17}$ - periodo segnato dall'esperienza delle sedute psicanalitiche ${ }^{18}$ - avesse riscoperto e portato a compimento la prigioniera ${ }^{19}$ e oscura vocazione del profondo.

Il tema della oscurità interiore come realtà sconosciuta e sommersa del poeta, prima dell'intervento liberante della psicanalisi, è ricorrente nella riflessione bauchauliana. Ora, se la domanda che Bauchau rivolge alla sua analista Blanche Jouve in primo luogo, ma a se stesso in seconda istanza: «Commencerais-je à sortir de l'obscurité où j'ai si logtemps vécu?», ribadisce l'importanza di questa idea, la risposta formulata dalla psicoterapeuta si carica di un significato nuovo e inatteso per Bauchau, rilevante per il lettore:

Je lui [scil. a Blanche Jouve] dis que l'analyse est un acte héroïque où le courage est d'autant plus grand qu'il ne se voit pas et que l'analysant apparaît faible, apeuré, humilié au moment même où il accomplit dans la durée l'acte essentiel de sa vie. Elle approuve. «Votre roman est une revanche, dit-elle, une revanche sur l'humiliation de l'analyse et l'obscurité où elle s'est passée $[\ldots] \gg^{20}$.

l'oscurità, del resto, è ricorrente nel Journal d'Antigone. Cfr. in merito JA, p. 207 , alla data 28 gennaio [1993]; p. 254, alla data 21 giugno [1993]; p. 333, alla data 20 giugno [1994]; p. 348, alla data 17 agosto [1994]. Cfr. anche EC, pp. 3-4.

(15) Cfr. EC, p. 4 (la sottolineatura è nostra). Lidea dell'affiorare della scrittura per mezzo della psicanalisi è enunciata anche in GM, pp. 35-36, alla data 3 marzo [1960]: «Aussi est-ce autour de l'analyse que se centre le récit. L'analyse fait ressurgir le passé, le transforme et lui donne une physionomie et une signification nouvelles. Le passé continue, à partir de là, à vivre et à évoluer. Le temps passé est aussi un temps qui revient "modifié", un temps qui arrive».

(16) Cfr. H. Bauchau, Introduction a GM, p. 11 [datata gennaio 2005] «Avant ce roman [La Déchiru$r e$ ] j'avais écrit des poèmes et composé une pièce de théâtre, j'étais déjà un écrivant, je n'étais pas encore un écrivain. Je n'avais pas encore confié à l'écriture l'opacité, la ténacité, l'espérance de ma vie». Ė noto che il riconoscimento letterario cui abbiamo accennato giunge, come Bauchau dichiara apertamente, in una fase avanzata della sua vita: «[...] je crois que pour comprendre mon parcours et mon aventure d'écrivain je ne dois pas oublier que j'ai été un athlète. Il m'a fallu attendre d'avoir soixante-dix ans pour être reconnu comme écrivain» (JA, pp. 142143 , alla data 23 febbraio [1992]). Per una ricostruzione della carriera poetica di Bauchau e della sua vicenda letteraria cfr. A. ScHмITZ, Henry Bauchau au risque de la presse, in AA. Vv., Henry Bauchau. Un écrivain, une aeuvre, cit., pp. 453-487.

(17) Cfr. EC, p. 6: «J'ai donc commencé vers 1948, 1949, au cours de mes années de ténèbres, à écrire des vers. Ces vers me résistaient, ils refu- saient de s'assembler en texte. Ils brillaient quelques instants d'un éclat furtif mais repoussaient l'articulation du poème et l'expression d'un sens. Si je m'obstinais, ils prenaient corps parfois, mais comme des poèmes brisés, déchirés avant de naître par une écrasante ou mutilante machine intérieure». Cfr. anche Rencontres avec Freud in EE, p. 136.

(18) Sulla natura liberante della psicanalisi e sul suo ruolo nell'innesco dei processi che hanno permesso all'autore di ricuperare la sua dimensione poetica egli insiste anche in altri scritti. Valga come ulteriore esempio quanto Bauchau dice l'otto agosto del 1992, prima della pubblicazione de L'écriture à l'écoute: «[...] il m'a fallu, quant à moi, tout le temps qui va de 1939 à la fin de mon analyse, à mon remariage et à l'écriture de Gengis Khan en 1954-1955 pour que je commence à m'approcher de l'écrivain que, sans le savoir vraiment, j'espérais être et que j'étais. Seize ans en somme pour entamer cette nouvelle vie, si follement fuie dans des égarements nécessaires. Et cette fuite, tout en diminuant d'importance, n'a pas cessé» (cfr. JA, p. 167).

(19) Vocazione prigioniera in Bauchau ancora fanciullo, castrata da un ambiente familiare per nulla avvezzo alle aspirazioni letterarie, considerate cose di poco conto, per gente superficiale alla ricerca di divertimento. Egli cresce, infatti, in un contesto radicalmente ostile alla figura di poeta, alle sue funzioni, ai suoi riconoscimenti sociali e proprio in questa avversione egli isola e identificherà più tardi, anche con l'aiuto della psicanalisi, il suo allontanamento da una passione come quella per la scrittura (cfr. JA, pp. 359-360, alla data 5 ottobre [1994]). Su questo tema cfr. anche $E C$, p. 26.

(20) GM, pp. 19 e 20, alla data 27 gennaio [1960]. 
In questo senso infatti il romanzo assurge a strumento di rivalsa sull'umiliazione subita nella fase psicanalizzante, ma anche sull'oscurità che al momento dell'analisi permea la realtà dell'autore. Pertanto la scrittura non è solo un modo per rivalersi della fragilità esistenziale che emerge dalle sedute di psicanalisi, ma è anche momento di riscatto in quanto ricupero di quella fragilità che attraverso l'atto di scrittura si impone come atto di forza, come «acte héroïque» appunto. Ma la scrittura, in particolare la poesia, si configura anche come strumento di rivalsa fra Henry e il fratello maggiore Jean. Strumento che, del resto, è necessario al ricupero di sé, di quella totalità e unicità dell'essere che se da un lato assume la propria forma attraverso il distacco dal primogenito, Jean, dall'altro tenta di impossessarsi di quel Tutto da cui ogni cosa è generata:

[...] Olivier [il fratello Jean che nella finzione letteraria assume questa identità] a suscité en moi pendant nos années d'enfance et de rivalité le sentiment d'être de trop. C'est contre lui que se rebelle le poème de la petite enfance qui étincelle quelques instants sous la forme illuminée du sabre. Tenant le sabre, tenant la plume, j'ai une arme pour faire face à l'opacité du monde [...]. Il écrira dans la terre et dans les lignées d'animaux, tandis qu'à mes risques et périls je devrai féconder et rendre obscure la page blanche ${ }^{21}$.

È interessante evidenziare - da quella che potremmo definire la lenta evoluzione della teoria poetica dell'autore - il rapporto dialettico e contiguo che si stabilisce fra i quattro poli (1. poesia; 2. infanzia; 3. fratello; 4 . Henry), intorno a cui si scandisce la fase prima della sua elaborazione teorica. Questi motori sono, per così dire, veicolanti un flusso creativo 'primordiale' e successivo che, concretizzato nella materialità oggettiva della poesia, ricupera il periodo dell'infanzia nel rapporto antagonista con il fratello, per giungere poi alla determinazione del 'sé-Henry' nella dimensione della scrittura. Il fratello Jean - Olivier nella ricostruzione narrativa appunto -, figura centrale nella vita e nella complessità dei dinamismi psichici di Bauchau, rappresenta la razionalità. Ora, proprio dal netto configurarsi della forza del razionale (= Olivier) si stacca, opponendosi e definendosi all'interno di una forma del tutto autonoma, il potere dell'inconscio, dell'irrazionale (= Henry), di quell'inconscio che va oltre e supera il limite persistente del raziocinio (la pesanteur du rationnel). Tale inconscio si imporrà liberamente come forza ed espressione poetica in un Bauchau ormai maturo, attraverso l'incontenibile energia della travolgente «immensité de l'inconscient», il cui potere stravolge e intimorisce. Il passo seguente è illuminante:

C'est dans cette lumière [luce che riflette la bellezza luminosa della poesia] incertaine que je rejoins l'enfance et sa circonstance éclatante. Car que représente l'image initiale du grand, de l'âné illuminé par le soleil et se balançant sur son cheval sinon, en face de moi, assis dans l'ombre au milieu des petits objets, l'efficace pésanteur du rationnel et l'immensité de l'inconscient. L'une écrase, l'autre fait peur. Cette image pourtant est à l'origine de mon œuvre, c'est elle qui a désigné les thèmes et les personnages qui m'ont passionné et leur a donné pouvoir sur mes livres. C'est ici que je dois situer ma poésie et mon écriture parmi celles qui sont nées dans ce que je voudrais appeler l'écriture après Freud ${ }^{22}$.

Il tema dell'oscurità, nel discorso sulla poetica, è strettamente connesso a quello della profondità. Anche l'idea della profondeur, infatti, come spazio interiore da cui è generata la scrittura permea alcune delle riflessioni teoriche bauchauliane ${ }^{23}$. Non a

(21) EC, pp. 3-4.

(22) EC, p. 16.

(23) Cfr. JJ, p. 113, alla data 16 ottobre [1985]:
«Après une journée prise par mes patients, je décide, presque par hasard, de reprendre mon texte le soir à 9 heures. Surprise: cinq pages surviennent, 
caso, questa impellente pulsione di creatività del profondo sarà indagata e ricuperata attraverso un processo di riaffioramento dall'inconscio dall'analista Blanche Jouve, colei che più tardi Bauchau definirà, probabilmente per questa ragione, la Sibylle. Essa lo sosterrà, incoraggiandolo nella ripresa dell'attività poetica da sempre presente nelle sfere più segrete dell'inconscio ${ }^{24}$. Bauchau ricorda questo importante momento della sua iniziale esperienza come poeta attraverso uno sforzo di memoria e di ricupero della sua storia, con un tentativo che pur filtrando il passato mediante l'uso del presente narrativo lo carica di una molteplicità di ricordi, i quali, inevitabilmente, tendono a proiettare l'autore e il lettore non nella immediata contemporaneità ma nel trascorso di un vissuto piuttosto lontano:

Malgré le manque de temps et une sourde mais constante dérision intérieure, je continue à écrire des poèmes. Je suis soutenu par une parole de celle que je n'appelle plus mon analyste, mais la Sibylle. Elle me demande un jour quel est pour moi le point fixe, le levier de l'analyse? Je réponds: «la confiance» puis, comme elle se tait: «la volonté de faire confiance». Elle ne me contredit pas mais, après un moment de silence, constate: «votre levier c'est l'écriture» [...]. La parole de la Sibylle prend une acuité singulière. Elle confirme la confiance de celle qui est alors mon unique lectrice et qui accorde à mes poèmes une attention et une importance qui me forcent, malgré mes résistances, à me reconnaître et à exister à mes propres yeux comme poète ${ }^{25}$.

Dagli esordi dunque di quella che sarà natura proteiforme di scrittore - ma ancor più di poeta, poiché questa è la veste che egli sperimenta agli albori della sua creatività - scrittura e psicanalisi sembrano profondamente intrecciate in una fitta messe di interrelazioni che, se affiorano nella loro complessità già da questa prima dichiarazione, emergono anche da un'attenta lettura della produzione teorica bauchauliana, nonché da plurime dichiarazioni dell'autore disseminate qua e là, soprattutto negli scritti autobiografici. Infatti, come in parte si è detto, è Bauchau stesso che isola la centralità del ruolo assunto dalla psicanalisi all'interno del suo laboratorio poetico, tanto che, in più frangenti, si sofferma con insistenza sulle salde e inconfutabili connessioni che intercorrono fra questo laboratorio compositivo da un lato, e l'universo psicanalitico dall'altro. Del resto, l'insistenza, l'enfasi e le iperboliche iterazioni di talune riflessioni, soprattutto quelle di cui è intrisa una raccolta di saggi dai contenuti perlopiù autobiografici ripubblicata di recente e qui già menzionata (L'Écriture à l'écoute), metterebbero in grado, anche il lettore più ignaro delle problematiche concernenti l'elaborazione poetica di Bauchau, di isolare immediatamente questo binomio fondamentale - poésie e analyse - intorno a cui essa costantemente gravita:

[...] Vous vous étonnerez peut-être de me voir, parlant de la genèse de ma poésie, parler autant de l'analyse. C'est que dans ma vie l'écriture et l'analyse se sont intimement liées. L'une a libéré l'autre ${ }^{26}$ et toutes deux ont continué à agir et à évoluer ensemble. L'analyse a été la coupure, l'étape décisive de ma vie. Il y a celui que j'ai été avant elle et dont je regrette souvent l'assurance, les certitudes et ce qui me semble maintenant l'ignorante innocence. Il y a celui qui

non sans scories mais avec un mouvement venu du tréfond»; e poi ancora: «Je sens constamment l'histoire d'Edipe et d'Antigone remuer au fond de moi, je ne trouve pas le temps de m'y plonger comme il le faudrait» (ibid.) e ancora: «Dans ce livre [scil. Edipe sur la route], j'ai vraiment labouré mes profondeurs» $(J J$, p. 141, alla data 30 maggio [1986]).

(24) Molto più tardi, il 2 luglio del 1998, di lei dirà: «Blanche a été le maître qui m’a orienté vers plus de lumière et d'exigence envers moi-même. Je ne sais pas ce que je serais devenu sans cette rencontre» (PBG, p. 74).

(25) EC, p. 9.

(26) L'idea secondo cui analyse et écriture vivono di un rapporto liberante - e in particolare l'insistenza sull'idea dell'analisi come esperienza che libera la scrittura e i processi di elaborazione poetica - è ulteriormente ribadita: «Ma première analyse a libéré l'écriture et quand je l'ai terminée prématurément j'ai pu la continuer en écrivant des poèmes et mon journal» (La scène du rêve in EE, p. 127). 
est après et dont l'univers intérieur a été labouré, transformé par l'expérience de l'inconscient et la découverte des terres inconnues de mon être ${ }^{27}$.

Così, se nel passo riportato, in particolare, Bauchau accentua con una certa enfasi due realtà (écriture e analyse) che si configurano come realtà inscindibili, questo stesso passo sembra sottolineare il rapporto dialogico («l'une a libéré l'autre») e di necessaria reciprocità nello scambio dell'una rispetto all'altra, proprio all'interno dell'itinerario poetico or ora tratteggiato. Questo itinerario, costruito appunto su due motori fondanti - poésie (= écriture) e analyse -, potrebbe essere identificato prima nel passaggio dall'analisi alla scrittura, poi nell'atto liberante che la prima esercita sulla creazione poetica, con la quale, peraltro, le «experience[s] de l'inconscient» si fondono e confondono in un ciclo metamorfico-generativo, ora nato dallo stemperarsi dell'una e dell'altra in un nuovo processo («et toutes deux ont continué à agir et à évoluer ensemble»), ora pronto a convergere nuovamente nella scrittura.

Tuttavia, in quello che è atto di creazione poetica, l'esperienza di analisi sembra prendere rilievo sulla pratica di scrittura, nella misura in cui un ulteriore intreccio pare affermarsi all'interno di questo ciclico processo dialogico, cioè quello sussistente fra écriture e analyse. Questa volta, dunque, non si tratterebbe più di una interconnessione tra scrittura e analisi, quanto piuttosto di una sotterranea interazione tra analyse e vie $e^{28}$. In tale prospettiva e nel coagularsi dell'existence nell'esperienza psicanalitica, e per il tramite di essa deve infatti essere letta questa stessa esperienza che si configura come «étape décisive de [la] vie [de Bauchau]» in quanto anello imprescindibile dalla coupure, nel cui solco stanno da un lato «celui qu'[il] a été avant elle», dall'altro «celui qui est après». In questo senso, se per certi versi l'esistenziale si fonde nella sperimentazione diretta della psicanalisi, esso si condensa anche all'interno dei reconditi meccanismi governanti la scrittura in nuce, quando necessita cioè di essere liberata dal lavoro di indagine sull'inconscio, secondo un'assoluta e apparente simultaneità di dinamismi elaborativi interni, che finirebbero con l'annullare i micro scarti cronologici che se oggettivamente legano da un lato, separano dall'altro, per frazioni di secondi, i convulsi movimenti strutturanti l'iter verso i cui sentieri pare essere convogliata la creazione poetica bauchauliana prima di essere oggettivata in scrittura.

Inoltre, da ciò che l'autore afferma con assoluta trasparenza formale, risulta evidente quanto l'esplorazione dell'inconscio abbia cambiato e interiormente sovvertito la sua vita, scissa in due vies: la prima identificata nell'esistenza di un uomo coronata di certezze - quelle stesse «certitudes» che appariranno in seguito come «ignorante innocence» -, la seconda invece in quella di un uomo diverso, di «celui qui [...] a été labouré, transformé» e che forse, al seguito della metamorfosi subita, si pone come vita di un individuo più complesso del precedente, forse più tormentato e inquieto poiché privo delle false certezze di un tempo («il y a celui que j'ai été avant [scil.

(27) EC, p. 9 (le sottolineature sono nostre). Lo stupore generato dalla scoperta di mondi sommersi, cui è possibile approdare attraverso l'esperienza della psicanalasi che mette in atto il cambiamento radicale dell'uomo e del poeta, è in qualche modo rafforzato dalla convizione che opera valida è quella originata proprio dalle profondità dell'inconscio: «[... Les choses bonnes que j'écris ne viennent pas du moi conscient, mais des profondeurs $[\ldots] \gg(J A$, p. 466, alla data $1^{\circ}$ settembre [1996]).

(28) Per un'ulteriore esemplificazione sui nessi che intercorrono fra scrittura/analisi, analisi/vita e vita/scrittura cfr. $J A$, p. 40 , alla data 14 giugno
[1990]; pp. 80-81, alla data 8 febbraio [1991]; p. 85, alla data 10 marzo [1991]; p. 177, alla data 28 agosto [1992]; p. 194, alla data 30 novembre [1992]; p. 211, alla data 12 febbraio [1993]; p. 348, alla data 17 agosto [1994]; p. 377, alla data 5 febbraio [1995]; p. 405, alla data 28 maggio [1995]; p. 416, alla data 11 agosto [1995]; p. 437, alla data 5 dicembre [1995]; p. 466, alla data 31 agosto [1966]. Cfr. anche JJ, pp. 251-252, alle date 23 luglio [1988] e 26 luglio [1988]. Cfr. poi EC, pp. 9-10; p. 20; p. 55; e l'intervento conclusivo (Chemins d'errance) in EC, pp. 60-80; in particolare, cfr. ibid., pp. 60-63. 
l'analyse] et dont je regrette souvent l'assurance, les certitudes et ce qui me semble maintenant l'ignorante innocence»).

Quindi, se l'elemento psicanalisi emerge e si impone come momento consustanziale della creatività bauchauliana, l'inconscio, ma soprattutto «l'expérience de l'inconscient», sembrano essere determinanti per quel processo di intima e travagliata trasformazione di cui dicevamo («il y a celui dont l'univers intérieur a été labouré, transformé»), che avrebbe favorevolmente indotto l'autore ad approdare a «terres inconnues de [son] être» ove lo stupore per il meraviglioso della «découverte» cela e svela ad un tempo una nuova incalzante realtà interiore, quella che probabilmente si configurerà come impulso creativo nella scrittura e discernimento di realtà, quali solo la scrittura ha il potere di disvelare. La poesia - giacché di essa qui vogliamo trattare - è allora quella realtà che ha origine da un meccanismo elaborativo del profondo, il cui anello generante però non deve essere riconosciuto esclusivamente nell'importante dinamismo sussistente fra scrittura e analisi da un lato e analisi e vita dall'altro, né tanto meno nel ruolo, pur determinante, che la psychanalyse, dunque l'inconscient, hanno assunto per il nostro autore. In questo senso è possibile sostenere che l'elaborazione creativa da cui scaturisce e prende forma la poesia, non sia in Bauchau da considerarsi come il solo risultato di un processo oggettivato dall'esperienza psicanalitica e dal ruolo dell'inconscio, e che essa non sia soltanto il frutto di un «mouvement $^{29}$ inconscient de la profondeur ${ }^{30} \gg$ tradotto in scrittura secondo implicazioni

(29) Il tema del mouvement è ricorrente negli scritti di Bauchau. Anche in questo caso egli si sofferma su tale concetto e puntualizza ciò che il 'movimento' significa in scrittura e le ragioni del suo configurarsi all'interno dell'atto creativo: «[...] je ne puis écrire que ce qui s'est d'abord intériorisé en moi. Ce n'est pas moi qui vais vers le poème, c'est lui qui vient vers moi. Cela commence par un son, un rythme, une image et j'ai soudain le désir, l'espérance d'écrire un poème. Je ne sais d'où surviennent ces sensations inattendues, je vois seulement qu'elles sont en mouvement et que, pour les retenir, je dois me faire mouvant comme elles» (EC, p. 14). Sempre sull'immagine del movimento, come momento essenziale e connaturato al processo di scrittura, cfr. JJ, p. 17, alla data 12 febbraio [1984]: «J'avais intitulé mon article La concordance des temps. Je dois chercher un autre titre, car celui-là a déjà été utilisé. Je m'arrête au mot 'connivence' et propose à Conrad La connivence des temps. Finalement Monique Schneider, consultée, trouve que La connivence des temps est juste et correspond mieux au texte. Quelques personnes ont lu l'article. Elles ont été sensibles aux idées que j'exprime et aussi au mouvement que je leur imprime dans le texte et qui est pour moi, avec le soin de voix, ce qu'il y a de plus important dans l'écriture». Cfr. anche JJ, p. 113, alla data 16 ottobre [1985]; pp. 114-115, alla data 29 ottobre [1985]; p. 151, alla data 28 luglio [1986]; p. 169, alla data 26 settembre [1986]; p. 203, alla data 14 agosto [1987] e $J A$, p. 467, alla data 2 settembre [1996]: « [...] j'ai écrit ce poème dans un mouvement de sensations et de mots»; cfr. anche $J A$, p. 12, alla data 19 settembre [1989]; p. 96 , alla data 18 giugno [1991]; p. 114, alla data 30 agosto [1991]; p. 333, alla data 20 giugno [1994]; p. 345, alla data 8 agosto [1994]; p. 467, alla data 2 settembre [1996]; GM, p. 34, alla data 26 febbraio
[1960]; p. 63 alla data 24 maggio [1960]; p. 74, alla data 27 luglio [1960]; p. 144, alla data 5 agosto [1961]; p. 158 , alla data 8 ottobre [1961]; p. 162 , alla data 28 ottobre [1961]; p. 175, alla data 4 dicembre [1961]; p. 198, alla data 12 maggio [1962]; p. 218, alla data 24 luglio [1962]; p. 386, alla data 23 marzo [1965]; p. 387, alla data 30 marzo [1965]; PBG, p. 27, alla data 20 settembre [1997]; p. 86, alla data 3 novembre [1998]; p. 357, alla data 8 luglio [2001].

(30) Il profondo, come luogo della genesi creativa, è metafora a cui Bauchau ricorre abitualmente: del profondo egli sottolinea soprattutto il ruolo, all'interno di quella elaborazione che - come abbiamo già detto - non può essere isolata dalla funzione esercitata dalla psicanalisi. Attraverso questo profondo, appunto, l'autore ricupera la sua natura di poeta e di scrittore in genere. A tale proposito cfr. $J A$, p. 40, alla data 14 giugno [1990], ove Bauchau ricorda di aver parlato alla sua analista di un progetto concernente la stesura di un romanzo. Egli annota infatti: «[...] je me rappelle en avoir parlé à Blanche et elle s'était intéressée à ce projet. Avant de parvenir à des zones plus profondes il faut, je le sais bien, que j'exprime encore d'autres choses $[. ..] \gg$. Su questa stessa immagine cfr. anche $J A$, p. 466, alla data 1 settembre [1996]; EC, pp. 5 e 7 . Il tema della profondeur però non è solo evocativo della realtà da cui nasce e si alimenta la scrittura, ma è anche sinonimo di ricupero di una dimensione esistenziale, di un raccoglimento intimistico che qualifica in modo totalizzante la vita dello scrittore, visto come colui che continuamente e ossessivamente anela a tutto ciò che è profondeur e a tutto ciò che quest'ultima rappresenta: «Il faudrait un retour au recueillement, à la vie profonde et à l'écriture [...]. Après Paris et la traversée de la France où tout était large, vert, torride et ensoleillé c'était le retour aux vraies 
anche esistenziali, quanto piuttosto il prodotto ultimo di un elaborato meccanismo ad incastro plurimo che rimanderebbe alle complesse dinamiche delle realtà compulsive dell'incoscient, ma parallelamente anche a quelle del conscient:

[...] c'est en vain que je voudrais capter le cri d'existence des voyelles. Ce qui était dictée abrupte, langage absolu, forme déjà plus que des sons dégonflés dans ma bouche. Quelque chose a voulu parler à travers moi mais il eût fallu une écoute plus fine, une main plus exercée que la mienne. Je ne suis toujours que le traducteur infidèle ${ }^{31}$.

In questo frangente infatti, quanto Bauchau asserisce - rivisitando e adattando così un topos letterario fortemente sfruttato - sembra sì denunciare la sua funzione di intermediario tra una realtà trascendente, convogliata forse all'esprit poetico attraverso i canali dell'inconscio («ce qui était dictée abrupte, langage absolu»; «quelque chose a voulu parler à travers moi») e una realtà contingente inserita in una parcellizzata fisicità per nulla trascesa ma contestualizzata all'interno di un cavo corporeo, che nulla ha di oltre-mondano («des sons dégonflés dans ma bouche»), ma attraverso tale denuncia egli esaurisce la sua enunciazione in due sintagmi conclusivi, ove i due ultimi lessemi («traducteur infidèle») ci sembrano riflettere un'enfasi e un semantismo rilevanti, a sostegno di quanto dicevamo circa il ruolo che inconscient e conscient detengono in Bauchau.

Ora, la particolare natura del poeta, di colui che è portavoce, «traducteur» appunto, di realtà metafisiche - natura che per certi aspetti richiamerebbe quella di poète voyant teorizzata tra la fine del Romanticismo e il consolidamento del Simbolismo - in Bauchau è traduttrice di talune realtà trascendenti, ma traduttrice infidèle. Questo dato, a nostro avviso non trascurabile, ci sembrerebbe riconducibile più al côté conscient dell'autore di quanto non sarebbe al côté attiguo, ma inverso, di inconscient. Pertanto, la presenza del conscio all'interno del processo di scrittura bauchauliano troverebbe ragione di essere proprio nella esplicita denuncia dell'infedeltà («infidèle»), di quell'elemento che tenderebbe cioè a variare o, comunque, a perturbare l'impulso creativo di derivazione inconscia, a causa dell'intervento, questa volta conscio, del poeta sulla materia elaborativa («quelque chose a voulu parler à travers moi ${ }^{32}$ mais [...] je ne suis

dimensions d'une vie modeste, retirée, tout orientée vers la profondeur [Bauchau parla di ciò che lo colpisce nel suo rientro a Bergrösli]. Malgré mon désir profond de m'en évader c'est ma vie, c'est le lieu où je dois œuvrer aujourd'hui et l'angoisse éprouvée à chaque retour, c'est la peur de reprendre le fardeau. Il faut renouer le fil interrompu, retourner dans la profondeur, retrouver l'univers romanesque plus vrai que la vie» (GM, pp. 128-129, alla data 4 luglio [1961]). Sul tema del raccoglimento intimistico cfr. anche GM, p. 321, alla data 5 dicembre [1963].

(31) EC, p. 7.

(32) Ė innegabile che la creazione poetica di Bauchau continuamente oscilla fra il côté inconscient et il côté conscient, con ribaltamenti di ruoli e di priorità riconosciute ora all'uno ora all'altro versante. Tuttavia, nonostante l'insistenza sulla dimensione inconscia come elemento fondante dell'elaborazione poetica, il ruolo del conscio quale anello di congiunzione indispensabile per la realizzazione di tale elaborazione, talvolta è riconosciuto dall'autore - anche in altra sede - proprio attraverso l'insistenza che mette in rilievo, nonostante tutto («cependant...»; «seulement...»), il ruolo dell'io narrativo («c'est moi...») come intermediario di una razionalità assolutamente e volutamente conscia: «j'ai écrit ce poème [Bauchau parla di quell'unico poema scritto nel corso di un anno. Si tratta del 1996] dans un mouvement de sensations et de mots. C'étaient les mots qui pensaient pour moi en cherchant soit à apparaître soit à prendre forme dans l'écriture. C'est moi ensuite qui, dans les mots du poème, ai reconnu les pensées qui me traversaient d'une façon libre, errante, qui n'était pas celle de la conscience à laquelle cependant, pour devenir poème, il fallait que les mots, non sans douleur, accèdent» ( $J A, \mathrm{p}$. 467 , alla data 2 settembre [1996]). Oscillazione che si configura spesso come apparente negazione del razionale per assurgere qui alla dichiarata priorità dell'irrazionale, in altri contesti però non riconosciuta come tale: «la poésie a sa source avant la pensée. La pensée vient seulement la joindre» ( $J A$, p. 28 , alla data 5 febbraio [1990]). Su questo punto cfr. infra la nota 33 e JJ, p. 292, alla data 31 luglio [1989]. Su quella che potremmo definire una fluttuazione di intenti critico-speculativi relativamente al dialogico del conscio e dell'inconscio rimandiamo alle pp. 342-350 di questo studio. 
toujours que le traducteur infidèle») $)^{33}$. Materia che sarebbe dunque la derivante di una remota dimensione sovrasensibile, ora riflessa e traslata, da cui in seguito essa erediterebbe una natura nuova, non più fissata nella forma da un «langage absolu» ma contaminata, proprio nella forma e probabilmente nei contenuti, dalla inadeguatezza del poeta, incapace per limiti umani di restituire in purezza, alla sua realtà, la bellezza assoluta del trascendente da cui attinge.

Questa idea del linguaggio assoluto richiama, per certi versi, l'idea dell'assoluto nell'uomo, riscattato dal peccato e chiamato alla vita dalla parola creatrice di Dio. La richiama ma la capovolge, poiché la realtà umana si configura come realtà opposta a quella divina. Il parallelismo che si può delineare - seppur rovesciato, poiché e contrario si configura la realtà creaturale rispetto alla natura divina - concerne così l'idea che, come la parola di Dio chiama alla vita dalla non vita, e come questa stessa vita a cui è chiamato a rispondere immette l'uomo in una condizione nuova di eternità perché essa è libera dal peccato, allo stesso modo il poeta cattura da una dimensione altra, assoluta, eterna, le voyelles, la loro stessa essenza immanente, tramutandola però in esistenza contingente. In definitiva, il poeta chiama alla vita il linguaggio che, antecedentemente, è «langage absolu», attribuendogli una natura corruttibile attraverso la forma che esso assume nella costrizione delle parole. Dio, l'Assoluto, chiama l'Essere alla vita dai morti, riscattandolo dalla morte del peccato, conferendogli natura incorruttibile e libera dai vincoli del corpo. In questo senso l'écriture, in Bauchau, sarebbe da intendere come la risultante di un «dialogue ${ }^{34}$ perceptible [...] entre la pensée consciente et la pensée incosciente ${ }^{35} \gg$ in un movimento circolare a struttura chiastica, ove una incessante tensione alternata fra conscient e inconscient appunto, spiegherebbe, ridefinendola, l'elaborazione poetica ora in nuce ora in atto del nostro autore.

(33) Circa una decina di anni dopo rispetto alle asserzioni contenute nella citazione, già riportata, di EC, p. 7 (cfr. supra, nota 31) Bauchau ritorna sulla posizione del poeta vis-à-vis della poesia o per meglio dire vis-â-vis dei reconditi meccanismi inconsci che regolano questo rapporto di elaborazione in cui, ribadisce l'autore, il poeta non resta che semplice traduttore chiamato a lasciare emergere il componimento che, in realtà, gli pre-esiste secondo natura autonoma: «Pendant que j'écris un poème, je découvre peu à peu ce qu'il est, ce qu'il est déjà alors que je n'en vois encore que des parties informes. Je m'efforce d'atteindre, de dévoiler cet inconnu et pourtant je sais que je n'y parviendrai pas tout à fait à cause des résistances accumulées en moi et dans la langue, qui m'empêchent de l'entendre dans son existence originelle. Je n'ai pas, je n'aurai jamais de lui une connaissance positive car le poète n'est pas le maître chez lui. Par attention à ce qui passe à travers moi, par écoute des rythmes de mon corps, par patience enfin, je puis parvenir à une approche négative du poème. Je pressens, j'entends ce qui n'est pas lui, les mots, les rythmes qui ne sont pas les siens et par découvertes successives je puis faire apparaître une certaine image, une certaine pensée, une part de la musique du poème appelé à naittre dont j'ai entendu l'appel. Je m'approche de sa circonstance, de ce qu'il est, mais je ne l'atteins pas, il m'éclaire de ses rayons, je ne suis pourtant que son traducteur, il ne vient pas de mon être et ne fera jamais partie de mon avoir car il n'est pas privatisable. Pendant tout le temps de mon travail je l'aimerai - même si souvent je le déteste -, mais mon désir lui restera étranger et sa langue me demeurera étrange. Le poète n'est pas maître chez lui car, pas plus que nous ne pouvons susciter nos rêves, nous ne pouvons diriger la profonde imagination» (L'innocence de l'oreille in EE, pp. 147-148). Sempre sulla preesistenza del poema Bauchau iniste particolarmente: «le poème est déjà là avant son écriture, on va vers ce qui existe et se dévoile peu à peu» (EE, p. 152), ma allo stesso modo egli ribadisce che lo scrittore non è creatore ma semplice traduttore: «[...] je concevais mon travail d'écrivain comme celui non d'un créateur mais d'un traducteur» (GM, p. 72, alla data 22 luglio [1960]).

(34) Bauchau insiste su questa immagine del dialogo interiore, al punto da apparire centrale a chi voglia tentare di ricostruire l'atto di scrittura bauchauliano, una volta che esso sia stato configurato e riconosciuto come espressione delle dinamiche interiori sottese al processo creativo stesso: «Quand j'écris dans Mélopée viking: "Les chevaux de la mer n'auront pas de poulains", vers donné, jailli directement des profondeurs, je projette dans l'imaginaire d'un passé ma crainte de voir l'inconscient cesser d'être fertile, cesser de me donner des poèmes à écrire au moment où l'espérance reprend courage et où je commence à m'ouvrir à la fécondité du dialogue intérieur» (EC, p. 10).

(35) Cfr. I. GABOLDE, Introduction, in EE, p. 12. 
Da questa interpretazione si potrebbe argomentare quindi che in Bauchau conscient e inconscient si richiamano senza sosta ogni qualvolta si tenti di ricostruire il dinamismo serrato che sottende i meccanismi intorno ai quali si costruisce la sua scrittura, poiché essenziale a quel 'circuito' di elaborazione poetica, spesso disorientante e pressoché vertiginoso, è certamente il gioco di interscambio fra queste due componenti.

Reciprocità e interdipendenza sembrano essere tratto caratterizzante il laboratorio di scrittura e l'universo poetico di Bauchau, teso - come dicevamo - costantemente fra l'uno e l'altro côté. Ma il nesso esistente fra la prima e la seconda realtà, contestualmente all'esprit poétique, per quanto innegabile, finisce talvolta con l'essere oggetto di riconsiderazione proprio rispetto alla natura delle due realtà suddette o per meglio dire all'equilibrio dell'una rispetto al ruolo dell'altra. Le dichiarazioni dell'autore sono in tal senso significative:

C'est hors du travail de la conscience que se font les véritables rencontres, découvertes et incendies de mots. La difficulté, insoluble le soir, se dénoue le matin parce que, sans que je le sache, 'quelque chose' y a travaillé toute la nuit ${ }^{36}$.

Così, se sembrava naturale - come abbiamo tentato di evidenziare poc'anzi - si potesse accogliere la tesi della imprescindibilità dell'inconscient rispetto al conscient, imprescindibilità fondante l'elaborazione poetica di Bauchau, intessuta di dinamismi impercettibili e delicati giochi di equilibrio, ora, questa stessa tesi sembrerebbe annullata e minata alle radici dal tono inequivocabile, quasi sentenzioso, che conferisce una nuova sfumatura semantica all'affermazione predetta. Infatti, la rilevanza attribuita all'inconscio dall'ultimo enunciato - costruito forse volutamente sui semantismi di rimando, tipici della retorica dell'implicito («c'est hors du travail de la conscience») - è paradossalmente resa dal lessema «conscience» la cui priorità, anzitutto sintagmatica, ci pare debba essere segnalata proprio all'interno di questo contesto. Priorità per certi aspetti solo apparente, poiché l'esplicita semantizzazione del passo «hors du travail de la conscience» rimanda invece - in prospettiva assiologica paradigmatica - alla necessità sottesa, lessicale e semantica, dell'antonimico della parola coscienza, l'incoscient appunto, proprio secondo quei giochi retorici di rimando cui abbiamo fatto cenno.

Ci sembra così necessario per il nostro discorso e per una priorità di costruzione argomentativa operare questo renversement di lessemi, proprio sul piano onomasiologico che, di rimando, si riflette su quello semasiologico. Il significante «conscience» è infatti investito di una centralità solo apparente. Esso è certamente mediato dai canali stilistici della litote, ma giunge in realtà a veicolare una realtà semantica che nega il contenuto da esso esplicitamente reso, proprio nel rimando implicito al suo concetto opposto, cioè a quello di inconscient che in questa nuova prospettiva assumerebbe, di fatto, importanza maggiore se relazionato al ruolo assunto dal conscient.

In realtà però le evoluzioni teorico-estetiche successive ci paiono riconducibili alla loro matrice generativa, quella cioè da cui eravamo partiti - concernente, lo ricordiamo, l'imprescindibilità del fenomenico dell'inconscio rispetto al fenomenico del conscio - e che in qualche modo abbiamo tentato di teorizzare. Pertanto, in questa direzione, l'assioma secondo cui mondo conscio e realtà dell'inconscio sono strettamente connesse e compenetrate, tanto da non poterne variare in nessun modo l'indivisibile co-natura, sembrerebbe ricuperato attraverso le parole con cui Bauchau chiude il discorso di apertura dell'Écriture à l'écoute: 
[...] le poème, dans l'écriture après Freud, est à la fois du côté de l'immense inconscient dont il procède et, traversant l'abîme qui les sépare, aux côtés du poète. C'est de ce double point de départ qu'il enfonce dans les ténèbres sa pointe acérée et brillante. Qui désigne ce qui est. Ce qui est à être ${ }^{37}$. Qui l'éclaire un peu. De ce peu qui ne suffit pas. Qui n'efface pas ce qui manque ni ce qui déborde. Et qui pourtant suffit, comme la grâce ${ }^{38}$.

$\grave{E}$ infatti in questa prospettiva, e a difesa di quanto precedentemente argomentato, che insistiamo su un passo della dichiarazione citata («le poème [...] est à la fois du côté de l'immense inconscient dont il procède et [...] aux côtés du poète»), ove risulta evidente il parallelismo fra il «côté de l'immense inconscient» e i «cotés du poète». Parallelismo che, se per certi versi pone l'accento sul ruolo esercitato dall'inconscio nella genesi creativa di qualsivoglia componimento poetico - peraltro secondo una concezione estetica che insiste sull'importanza del pensiero freudiano e post freudiano per la messa in luce dei meccanismi che governano la scrittura -, per certi altri ristabilisce equilibrio fra i poli centrali (inconscient/conscient) che agiscono come motori della scrittura bauchauliana, evidenziando ancora una volta il rapporto di dialogica contiguità dell'uno rispetto all'altro. Dalla riflessione conclusiva di Bauchau qui citata, proprio per l'evocazione della traversata dell'abime, fino a giungere «aux côtés du poète», noi possiamo ripercorrere idealmente l'itinerario artistico del nostro autore, che dalle impercettibili profondità del subconscio approda alle realtà del conscient, passando per il vaglio dell'intelletto e del raziocinio. In questo senso il sottile equilibrio di cui dicevamo è rimesso in gioco proprio attraverso l'insistere di Bauchau sull'idea di ciclicità, di incessante rapporto dialogico appunto, esistente fra inconscient e conscient, fra conscient e inconscient; idea ribadita dal riconoscere in essi non un punto di partenza, non un punto di arrivo, ma un «double point de départ», secondo una equilibrata attribuzione di ruoli, identificata nella reciprocità della loro perfezione simmettrica.

L'oscillazione tra realtà del conscio e dinamismi dell'inconscio - come abbiamo avuto occasione di ribadire - sono continue in Bauchau, come pure è mutevole, proprio nell'incertezza quasi delle dichiarazioni su e intorno a questi due motori della creazione poetica, la posizione dell'autore. Tuttavia, estendendo le sue riflessioni alla prosa e a ciò che conscio e inconscio rappresentano per il romanzo, Bauchau ricupera in pieno, ci sembra, il discorso argomentato a proposito della poesia quando, in una lunga digressione articolata in due giorni diversi, annota:

Hier soir, Conrad Stein me téléphone. Il a lu le manuscrit précédent de mon livre et me dit: «Il faut couper résolument tout ce qui est commentaire. Ton livre est un grand poème, il faut donc privilégier le poème, qui n'a pas besoin d'explications. Il faut aller plus directement au but». Cette conversation très amicale a été aussi très éclairante et m'a fait réfléchir. Je ressens cet entretien comme une poursuite, vingt ans après, de mon analyse avec Conrad. En me disant «Il faut privilégier le poème», il a fait une interprétation qui m'a permis d'entendre ce que je savais déjà sans parvenir à me le formuler. Il m'a montré que le conscient a voulu faire un autre livre que celui que désirait faire l'inconscient. Il faut revenir à l'initial'39.

Ma poi, sulla scorta del ricupero dell'idea di una perfetta simmetria tra i ruoli che conscient e inconscient esercitano nell'elaborazione poetica, il giorno seguente a queste prime riflessioni egli aggiunge:

(37) Per una interpretazione e per i rimandi lessicali e di immaginario insiti nel sintagma nominale «ce qui existe / ce qui est» cfr. supra la nota 33 di questo studio. Cfr. inoltre L'écriture et la circonstan- ce, cit., p. 72 .

(38) EC, p. 17 (le sottolineature sono nostre).

(39) JJ, p. 292, alla data 30 luglio [1989]. 
Ce n'est pas l'inconscient qui a surchargé mon livre de ce trop que je m'efforce d'éliminer. C'est le civilisé, au contraire, qui le lui a infligé par son besoin de comprendre. Il y a sans doute eu là une erreur nécessaire. Lénine: «Nous n'avons pas besoin des élans hystériques mais, au contraire, de la marche cadencée des bataillons de fer du prolétariat». Toutes les révolutions, y compris les révolutions intérieures en chacun de nous, sont traversées par ce conflit. Si l'un des deux mouvement l'emporte, la catastrophe ou l'enfermement dans des mourailles de fer ne sont pas loin. Il faut vivre ce conflit, laisser vivre les deux pulsions $[\ldots]^{40}$.

Questo processo elaborativo, che potremmo definire metamorfico ri-generati$\mathrm{vo}^{41}$, visto che la sua essenza pare proprio essere quella tipica di un ciclo autogenerativo costantemente rivitalizzato - lo stesso che sarebbe possibile isolare nella dinamica di creazione e di ri-creazione della materia, qui estendibile alla parola che genera e rigenera se stessa -, potrebbe essere identificato nel ritorno del poema alle sue origini «[le poème] enfonce dans les ténèbres sa pointe», là dove le «ténèbres» potrebbero essere assunte come il luogo della materia verbale ancora informe, la poetica in nuce, la cui espansione prima e creazione poi nella parola necessitano appunto del «cri d'existence des voyelles» ${ }^{42}$.

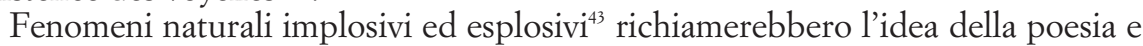
dell'opera d'arte in genere come espressione di realtà che emergono con forza dirompente dalla materia. L'idea di creazione e di ricreazione di questa materia, oggetto della poesia, per il tramite dei processi creativi di elaborazione e rielaborazione continue, è presente - in riferimento questa volta alla prosa - anche in alcune annotazioni contenute nel Journal d'Antigone ${ }^{44}$. Il tutto naturalmente in parallelo a una primissi-

(40) JJ, p. 292, alla data 31 luglio [1989] (le sottolineature sono nostre).

(41) Una sorta di definizione di questo processo e dell'elaborazione poetica in genere si trova in $J A$, p. 392, alla data 12 marzo [1995]: «Pour accéder à l'art, il faut vivre le désir des pulsions mais, par une profonde et patiente métamorphose, le transformer et parfois le sublimer dans un autre mode d'être».

(42) Cfr. EC, p. 7.

(43) È infatti attraverso l'idea di esplosione, con lo sfociare di immagini che emergono dal ricupero di certe realtà naturali, che Bauchau dà rilievo ai fenomeni sotterranei che sottendono la creazione narrativa. L'immagine del vulcano in eruzione ci pare infatti particolarmente rappresentativa: «[...] La création romanesque en moi est poussée en profondeur par le désir d'écrire un roman consacré à mon enfance et à ma jeunesse. Roman que je n'écrirai peut-être jamais mais qui me force à mettre au jour tout ce qui devait et doit encore naittre avant lui. C'est comme un volcan qui fait sortir de lui des couches successives de lave $[\ldots] \gg(J A$, p. 40, alla data 14 giugno [1990]: le sottolineature sono nostre).

(44) Cfr. JA, p. 345, alla data 10 agosto [1994]: «Hier, j’ai relu toute la dernière partie de la première version, celle qui a trait à la dernière marche d'Antigone et à sa mort. J'ai lu pendant une heure et demie car le texte est embrouillé parfois et l'écriture, souvent peu déchiffrable. C'est à nouveau une matière, avec des moments de chaos, mais il y a un mouvement, une vigueur pathétique, peutêtre exagérée. Si j’arrive à ordonner cette partie, à la resserrer en lui gardant sa qualité d'émotion et de vérité, ce sera peut-être un des moments de l'œu- vre» (i corsivi sono nostri). Cfr., sempre in merito all'idea di materia e della creazione di essa attraverso i processi elaborativi, alcune annotazioni che rievocano per certi aspetti la fenomenologia della creazione della materia universale. Rimandiamo in particolare a ciò che Bauchau afferma in una pagina del suo Journal d'Antigone a proposito del cahos primordiale, rappresentativo appunto del primo stadio della materia terrestre ancora informe: «La matière est là, une matière riche, mais très peu ordonnée, parfois proche du cahos. Je me suis senti un peu découragé aujourd'hui, en me disant que j'en avais encore pour deux ans» (JA, p. 343, alla data 8 agosto [1994]). Cfr. anche GM, p. 157, alla data 8 ottobre [1961]. È interessante notare come nei casi citati però l'idea di caos cui egli fa riferimento non rievochi solo lo stato generativo e ancestrale sottostante la creazione della materia universale, del mondo per intenderci, ma come essa possa anche rimandare allo stato di confusione, di vertige, di malessere che attanaglia l'autore in più frangenti, spesso legati alla redazione dei suoi scritti, in particolare alla fatica della redazione e della revisione continue. Cfr., soprattutto a questo proposito, JA, p. 181, alla data 6 settembre [1992]; p. 197, alla data 13 dicembre [1992]; pp. 200-201, alla data 11 gennaio [1993]; p. 202, alla data 13 gennaio [1993]; p. 204, alla data 17 gennaio [1993]; pp. 206-207, alla data 26 gennaio [1993]; p. 209, alla data 31 gennaio [1993]; p. 213, alla data 23 febbraio [1993]; p. 227, alla data 7 aprile [1993]; p. 229 , alla data 14 aprile [1993]; p. 235 alla data 13 maggio [1993]; p. 244, alla data 30 maggio [1993]; p. 249, alla data 13 giugno [1993]; p. 292, alla data 
ma lettura che, secondo archetipi noti a una tradizione letteraria plurisecolare, insiste invece sull'idea piuttosto abusata della poesia come luce di conoscenza che rischiara le tenebre dell'ignoranza. Tuttavia, se ricuperiamo quanto detto finora, rivisiteremo il nostro statuto di destinatari del discorso e ci collocheremo all'interno di una dinamica semantica nuova, proprio in quanto fruitori di un messaggio costruito non su una, ma su due immagini facenti capo a due diverse realtà: come dicevamo, quella della poesia e quella della materia. Del resto, la realtà referenziale cui rimanda la scelta dell'aggettivazione («acérée», «brillante»), ma anche la scelta di altri sintagmi («traversant l'abîme», «enfonce dans les ténèbres») è evocativa di quel genere di fenomeni naturali che giustificano l'evoluzione per gradi della massa informe in materia, come principio scientifico-razionale della creazione ${ }^{45}$.

\section{Linguaggio teologico e scrittura poetica.}

Ora, se da un lato Bauchau pare insistere sulle componenti fulcro che spiegherebbero la démarche della sua creatività, con aperture di immaginario implicanti l'idea della materialità universale (appartenente al campo del realisticamente conscio), dall'altro egli sembra invece rifarsi a un'idea di sacralità (appartenente al campo del profondamente inconscio), sacralità che investirebbe l'atto di creazione poetica, ma implicitamente, di rimando, anche quello concernente la creazione tutta ${ }^{46}$. Si tratta dunque, in Bauchau, di un registro plurimo del discorso: di poetica, di antropologia e di etica, nell'intersecarsi di linguaggio e di immaginario di derivazione genericamente teologica (più specificamente biblica, attraverso mediazioni tipicamente francesi, come quella pascaliana, ma forse anche quella di Claudel), senza che questo debba indurci a credere nella presenza di teologumeni estranei al militante psicanalista. Un testo come La Sourde Oreille - esemplare nel ricostruire l'itinerario di formazione e nello stesso tempo vero banco di prova di una scrittura densa di reminiscenze di natura eterogenea - offre testimonianza della presenza del linguaggio biblico all'interno del discorso psicanalitico:

La Bible était, pour ton ami, un grand torrent barbare, plein d'étincelles et de détresse et de prémonitions.

Il te lisait de longs passage d'Ezéchiel ou ces versets de la Genèse qui ont l'accent monumental des grandes pierres levées ${ }^{47}$.

19 settembre [1993]; p. 308, alla data 27 dicembre [1993]; p. 315, alla data 3 febbraio [1994]; p. 345, alla data 10 agosto [1994]; pp. 368-369, alla data 31 dicembre [1994]; p. 380, alla data 12 febbraio [1995]; p. 390, alla data 1 marzo [1995]; p. 425, alla data 27 settembre [1995]; p. 426, alla data 21 ottobre [1995]; p. 432, alla data 11 novembre [1995]; p. 434, alla data 24 novembre [1995]; pp. 475-476, alla data 15 novembre [1996]; pp. 496-497, alla data 13 maggio [1997]. Cfr. anche JJ, p. 94, alla data 22 luglio [1985]; p. 97, alla data 14 agosto [1985]; p. 111, alla data 11 ottobre [1985]; p. 151, alla data 28 luglio [1986]; p. 288, alla data 15 luglio [1989]; p. 289, alla data 18 luglio [1989]; pp. 290-291, alla data 26 luglio [1989]; p. 293, alla data 5 agosto [1989]; p. 294, alla data 9 agosto [1989]; p. 301, alla data 1 settembre [1989].

(45) Cfr. EC, p. 17 (testo citato supra).

(46) Proprio sulla creazione poetica e sulla crea- zione universale, ricondotta però a un principio giustificativo di matrice scritturale e non scientifico razionale Bauchau, alcuni anni prima della pubblicazione de L'écriture à l'écoute, offre una definizione che per certi versi conferma le riflessioni contenute nella raccolta di saggi citata, qui riportate: «À une réunion à Bruxelles, on m'interroge sur la création artistique et littéraire. Je réponds que, pour la première phase du processus tout au moins, le mot création est inadéquat. Il s'agit alors de laisser monter en soi ce qui vient d'une source inconnue et qui a sans doute son origine dans la toute petite enfance. La création, au sens de la Genèse, se fait à un second stade, lorsqu'il s'agit de mettre en ordre, de séparer ou d'établir des passerelles et des cheminements souterrains entre les différentes parties» (JA, p. 17, alla data 25 novembre [1989]).

(47) SO, p. 31. 
$\mathrm{Tu}$ as pris en sortant et sans savoir pourquoi, un livre de petit format. C'est l'Évangile. Selon quel saint? Selon saint Luc.

Ensuite ils sont tous passés, pendant longtemps sans lassitude. Selon aussi s'est éclairé, selon qui te donnait le son de l'Évangile.

Alors, par hasard croyais-tu ou en reponse à ce qui fut écrit par le rêve de Freud sur la table des profondeurs, tu as ouvert le livre $\operatorname{logie} e^{48}$.

Pour ton bonheur, pour ton malheur étroitement mêlés et pour l'animation de ta géo-

Qui me font voir, effaçant la banalité, me font entrer, avec ma voix et mes images, dans l'existence évangélique de l'oreille ${ }^{49}$.

[...] Oui, j'ai couru comme un voleur de feu en aspirant le monde dilaté où respiraient mes poumons raisonnables Salomon

En pensant aux chemins, aux puits de Galilée, au lys des champs plus beau que le roi

Qui s'est effacé doucement de sa tige. L'admirable et sexuel parfum n'est plus que dans les mots qui viendront pour le dire

Qui ne parleront pas de forme ni d'odeur mais qui diront: Considérez les lys des champs, comme ils croissent.

Et comment crôtre, comment aimer, comment croire autrement que dans l'épiphanie de l'amoureux parfum ${ }^{50}$.

Tu t'es arrêté sous un arbre, tu t'es couché au milieu des fougères. Leur forme, leur présence, sont à jamais liées aux mots de l'Évangile

Valga anche come esempio, nel brano dell'Écriture à l'écoute citato nel capitolo precedente ${ }^{52}$, la scelta di termini appartenenti al campo semantico della luce («l'abîme», «enfonce dans les ténèbres sa pointe acérée et brillante», «qui l'éclaire un peu»), che sembrerebbe costeggiare proprio alcune delle contrapposizioni luministiche che richiamano l'immaginario biblico ${ }^{33}$. L'identificazione della poesia con l'immagine della luce che brilla nell'oscurità delle tenebre funzionerebbe ancora una volta come eco di un'ermeneutica di ascendenza scritturale e si porrebbe anch'essa come la risultante di un immaginario poetico costruito anche sui poteri evocativi della parola, con implicazioni semantiche di matrice biblica, di cui il tessuto lessicologico bauchauliano è sicuramente intessuto. Queste scelte lessicali, infatti, potrebbero essere anche risonanze bibliche inconscie, riflesso che diventa eco scritturale, talvolta inconsapevole. Il che giustificherebbe un differente approccio ermeneutico ai testi di Bauchau in vista della ricostruzione di un discorso di poetica che fonde di continuo ascendenze concettuali diverse, implicanti il campo semantico scritturale e teologico.

Riprendiamo ancora una volta il segmento dell'Écriture à l'écoute: ivi Bauchau, evocando Freud quale matrice di concezione poetica ${ }^{54}$, sembra mescolare in memoria

(48) Ibid., p. 33.

(49) Ibid., p. 34.

(50) Ibid., pp. 34-35.

(51) Ibid., p. 35.

(52) Cfr. EC, p. 17 (supra, p. 348).

(53) L'immaginario della contrapposizione luce/ tenebre è ricorrente nella Bibbia: citiamo soltanto Isai., 9, 1; 42, 16; Jerem., 13, 16; Matth., 4, 16; Luc., 1, 79; Joan., 1, 5; I Cor., 4, 5; II Cor., 4, 6; 6, 14; I Joan., 2, 8-11.

(54) Cfr. anche $J A$, p. 40, alla data 14 giugno
[1990]: «La création romanesque en moi est poussée en profondeur par le désir d'écrire un roman consacré à mon enfance et à ma jeunesse. Roman que je n'écrirai peut-être jamais mais qui me force à mettre au jour tout ce qui devait et doit encore nâ̂tre avant lui. C'est comme un volcan qui fait sortir de lui des couches successives de lave. La plus proche du feu originaire est sans doute cette histoire de l'enfance et de la jeunesse jusqu'à l'analyse. Je me rappelle en avoir parlé à Blanche et elle s'était intéressée à ce projet». 
una filière di testi vulgati (perfino nelle antologie scolastiche) - dal Rimbaud della Lettre du voyant alle riflessioni teoriche dei Surrealisti - soprattutto per quanto concerne l'«enfoncement dans les ténèbres». Tuttavia, proprio in questo brano, ritroviamo un esempio interessante di intertestualità (in parte equivoca) con fonti di diverso ordine (in questo caso di ordine biblico e teologico) nella ripresa finale, a livello lessicale, del riferimento alla nozione paolina di grazia sufficiente ( $«$ Et qui pourtant suffit, comme la grâce»), nozione che nella storia della spiritualità francese, debitrice del grande débat teologico secentesco, ha una risonanza fortissima. In un punto di così grande pregnanza, l'eco del versetto paolino «Ma grâce te suffit» (2 Cor., 12, 9) non giustifica per certo - come non la giustifica il ripetersi di stilemi biblici e teologici nei testi di Bauchau - la lettura in chiave teologica del brano, ma avvalora l'ipotesi di una quanto mai attiva reminiscenza dell'autore di loci scritturali che costantemente emergono nel tessuto linguistico e ideologico delle sue riflessioni, imbevendolo di contenuti che oltrepassano le dinamiche sottese al discorso narrativo tout court e che fanno di esso il luogo privilegiato per una indagine parallela volta qui a ricostruire i pellegrinaggi spirituali e non solo gli itinerari discorsivi di Bauchau. Occorre peraltro sottolineare che l'uso equivoco o analogico di un riferimento biblico - come è il caso del citato versetto paolino nel passo dell'Écriture à l'écoute - non ne vieta in altri luoghi un uso rispondente al significato testuale. D'altronde, ciò che interessa al presente discorso per ricostruire il laboratorio bauchauliano di scrittura - occorre ribadirlo con chiarezza - è quello che abbiamo testé definito registro plurimo a livello di linguaggio, e non tanto i contenuti teologici veri e propri $\mathrm{o}$, più genericamente, di spiritualità.

Contenuti peraltro che esistono in Bauchau, come testimoniano anche i due Journaux (quello d'Edipe e quello d'Antigone). Basti pensare all'atto di fede nell'esistenza di Dio che si radica in un richiamo a Eckhart (uno dei grandi spirituels che ritornano nei testi bauchauliani, come sant'Agostino, Teresa d'Avila e Giovanni della Croce):

Maître Eckhart: «C'est chaque jour la plus grande fête, celle de l'existence de Dieu». Il y a dans cette petite phrase quelque chose qui me touche très profondément et à quoi, enseveli dans le quotidien comme je suis, il est bien difficile de faire face. Chaque matin je m'exerce au sourire en me répétant que Dieu est vivant. Je le vis en soulevant la fatigue pour affronter le jour neuf et il est vrai que cela comporte une mise en présence brève, un moment de sourire mais cela ne devient pas la fête de l'existence. Il y a une distance qui persiste entre le quotidien où souvent je m'enlise et la fête que je ne connais plus guère. Il se peut que Dieu ne me soulève pas car je ne compte pas assez sur lui pour cela. J'essaie de me soulever moi-même, souvent j'échoue, parfois je réussis un peu, mais je sais que de cette manière je ne puis aller ni très loin ni très haut ${ }^{55}$.

Basti pensare anche all'attenzione prestata al tema della preghiera (della preghiera cristiana):

Oui, la prière tient une grande place dans ma vie bien que je n'y consacre pas beaucoup de temps. C'est une prière chrétienne celle que je connais. Je ne suis jamais sûr de vraiment

(55) JA, p. 237, alla data 18 maggio [1993]. Pochi giorni più tardi, nello stesso journal, Bauchau riprende e insiste: «La pensée de Maître Eckhart: "C'est chaque jour le jour de la plus grande fête, le jour de la fête de l'existence de Dieu" s'intègre peu à peu dans ma vie. Dans mes exercices du matin mais aussi assez souvent dans la journée. Je voudrais en faire un poème mais il faut d'abord que je vive plus profondément cette pensée. Le jour de la fête de l'existence de Dieu me réjouit car c'est une fête, non pas une preuve. Il me semble pourtant aujourd'hui qu'aller vers le jour de la fête de l'existence c'est aller plus loin que vers la fête de l'existence de Dieu. Si, comme l'a dit Jacques Pohier, Dieu est Dieu et n'est donc pas tout, il y a place à côté de celle de l'existence de Dieu pour une fête de l'existence»: $J A$, p. 242 , alla data 29 maggio [1993]. 
comprendre les penseurs et spirituels indiens, chinois et musulmans que j'admire. Je vis et pense en chrétien du seuil et non en chrétien prétendument libéré. La prédominance de la morale et de l'organisation dans l'Église ne me permet plus de me sentir à l'intérieur. Je ne juge pas pour cela l'Eglise, c'est l'état où je suis.

Je pense chaque jour à la parole qui a été dite à saint Paul: ma grâce te suffit. Il l'a entendue pour lui mais non pour l'Église et son attitude envers le monde. Il est vrai que la vie un peu recluse en écriture que je mène depuis quelques années me pousse peut-être sans que j'en aie conscience à sousévaluer l'action, que je respecte pourtant et sais bien nécessaire $e^{56}$.

Riflessione sulla preghiera che si salda con una presa di distanza dall'istituzione Chiesa intesa come struttura organizzativa e in cui, per di più, lo psicanalista Bauchau denuncia la «prédominance de la morale» ${ }^{57}$. Riflessione che si àncora, nello stesso tempo, al testo biblico, come evidenzia il riferimento al brano paolino, riferimento che abbiamo già evocato, ma che appare un orientamento ossessivo della meditazione bauchauliana:

Chaque jour, depuis de nombreuses années, je me redis la phrase de saint Paul, lue sur une tombe au cimetière des vignerons aux Épesses: «Ma grâce te suffit». Cette pensée n'a cessé d'orienter ma vie et mon œuvre ${ }^{58}$.

Il nostro leggere, tuttavia, il discorso spirituale di Bauchau in funzione dei molteplici registri di un linguaggio che si realizza anzitutto nella creazione letteraria è giustificato dal nesso continuo che l'autore crea, nell'immedesimarsi con la sua opera, fra il livello esistenziale suo e il testo appunto, nesso mediante cui costruisce una rete di rapporti che vanno ben al di là dei giochi, retorici, della scrittura. Tale d'altra parte è il senso delle riflessioni che scandiscono i journaux, diari che sono praticamente sempre journaux di un'opera e non cronache autobiografiche di accadimenti esterni. Come si evidenzia qui, nel Journal d'Antigone:

J'avais en somme écrit avec courage, avec foi cette mort d'Antigone qui m'éprouvait tant. Mais la foi ne suffisait pas. Saint Paul l'avait fait remarquer avec justesse aux Corinthiens: «Quand j'aurais la foi qui soulève les montagnes, s'il me manque l'amour, je ne suis rien». Mon texte, mon brouillon, achevé, j'avais tenté d'occulter la mort d'Antigone au lieu de continuer

(56) $J A$, p. 219, alla data 15 marzo [1993].

(57) Bauchau ha avuto una giovinezza cattolicamente impegnata. A vent'anni pubblica un pamphlet sulla necessità di una politica cattolica sociale (Pour une politique catbolique. But, tendances, action, Bruxelles, Editions contemporaines, 1933), in cui critica con forza il clericalismo e mostra di considerare Maritain un punto di riferimento. Insiste sulla necessità di posizioni spiritualiste che si diversifichino da quella che egli definisce politica materialista e neutralista. Per certo gli aspetti politici, anche a livello teorico e organizzativo, prevalgono negli interessi del giovane Bauchau su quelli più prettamente religiosi. Si può comunque parlare di una formazione cattolica globale data la sua militanza fino agli anni della seconda guerra mondiale. Alcuni testi chiave (e non soltanto La sourde oreille ou le rêve de Freud o i due Journaux ora citati), che ripercorrono autobiograficamente gli snodi fondamentali dell'itinerario interiore bauchauliano, testimoniano di una forte presenza del riferimento alla spiritualità biblica e cattolica, anche al di là del- l'abbandono della comunione ecclesiale. «Cristiano sulla soglia» ama, d'altronde, definirsi Bauchau: «Dieu est vivant, je ne mets pas cela en doute même si curieusement je n'ai pas le désir d'en parler aux autres. Les Évangiles eux aussi sont vivants. Je ne me sens pas hors de l'Église en restant sur le seuil. Je respecte l'Église dans sa foi, sa mission de transmission et son espérance mais je la ressens comme trop intitutionnelle et affadissant la parole et la vie de Jésus. Je prie, j'écris, je porte mes petits fardeaux dont le plus lourd est le manque de temps»: $J A$, p. 227, alla data 7 aprile [1993]. Sulla formazione e sulla militanza cattolica di Bauchau è fonamentale A. NeuschäFER, De «La Cité chrétienne» au «Journal d'un mobilisé», in AA. VV., Les Constellations Impérieuses d'Henry Bauchau, cit., pp. 47-75.

(58) JA, p. 44, alla data 22 janvier [1996]. Ricordiamo ancora, per i riferimenti alla citazione paolina, JJ, pp. 127, alla data 1 febbraio [1986]; 169, alla data 25 settembre $1986 ; 245$, alla data 10 maggio [1988]. 
à la vivre, à l'aimer. Je ne voulais plus souffrir et c'est Antigone qui avait souffert en moi de cet homme sans amour «qui n'était rien», qui ne pouvait plus rien pour elle. Elle s'était révoltée, et m'avait révélé ainsi ma fragilité et celle du monde sans amour ${ }^{59}$.

Per quanto poi concerne la ricostruzione di una poetica con ascendenze, come si è detto, concettuali diverse, implicanti anche il campo semantico della spiritualità cristiana, se ci rifacciamo al discorso ermeneutico scritturale, possiamo essere indotti a cogliere l'eco di alcune riflessioni teologiche che circolavano - indubbiamente, qui, spesso usate con diverso segno - nell'Europa degli anni tra il sessanta e il novanta. Non si vuol dire che Bauchau sia stato interprete fedele degli autori di queste riflessioni (d'altronde non ha mai inteso d'esserlo!), ma semplicemente che le sue non ben definite tensioni al divino, alla verità dell'esistenza ${ }^{60}$, alla speranza di un'altra vita, alla luce «qui éclaire un peu l'abîme» ${ }^{61}$, risentono, consapevolmente o meno, del clima culturale di un'epoca sulla quale fortemente pesa una situazione storica, caratterizzata da sconvolgimenti di ideali individuali e collettivi.

Esemplifichiamo con un passo di Bauchau, cui si è già fatto riferimento in nota:

Dominique a trouvé L'Arche beau, mais un peu mélancolique. Elle a ajouté: mélancolique comme le temps, le temps qui passe. Autant qu'un poème sur le temps, L'Arche est, je crois, un poème sur l'espérance d'une autre vie, telle qu'on peut la ressentir dans cette époque où pèse sur nous la mort de Dieu, des dieux et des idéaux collectifs ${ }^{62}$.

È evidente che la nozione di 'morte di Dio' è talmente diffusa e volgarizzata negli anni sessanta ${ }^{63}$ che sarebbe non fondato basarsi su di essa per stabilire ascendenze o letture bene individuate. Ciò non toglie che la prospettiva critica di Bauchau riporti qui (ma anche in altri casi) a un'area di riflessione 'teologicamente' connotata. Come avviene per un'altra nozione evocata da Bauchau, quella della «faiblesse de Dieu», riecheggiata per di più attraverso un autore per certo non 'teologico', quale Marguerite Yourcenar:

Bref extrait d'une lettre de Yourcenar à une religieuse: «venir en aide à la faiblesse de Dieu». Cela rencontre tout à fait ce que j'éprouve au sujet de la faiblesse et du silence nécessaire de Dieu à notre époque. Il y a des époques où il a pu être ressenti dans sa gloire et sa puissance. Notre époque est celle de son retrait, de sa modestie. Pourtant, il y a des instants brefs où il ne peut s'empêcher de briller ${ }^{64}$.

(59) JA, p. 286, alla data 3 settembre [1993].

(60) Cfr. EC, p. 15 (il testo in questione è citato e commentato infra, p. 357).

(61) Cfr. EC, p. 17 (supra, p. 348).

(62) JJ, p. 103, alla data 2 settembre [1985].

(63) Non si fa qui riferimento ad archetipi filosofici ottocenteschi, quanto piuttosto a quel filone teologico, la cui matrice va ricercata in alcuni spunti bonhoefferiani, che negli anni sessanta diventa veramente (e uso la formula in senso riduttivo) alla moda con autori quali Harvey Cox, William Hamilton, Paul van Buren, Gabriel Vahanian e altri: cfr. B. Mondin, I teologi della morte di Dio, Torino, Borla, 1968. Ricordiamo anche che l'opera e il pensiero di Bonhoeffer (essenzialmente per quanto concerne la volgarizzazione della nozione di «morte di Dio») sono resi di dominio europeo - al di là della cerchia ristretta degli specialisti - da un libro-pamphlet, del 1963, del vescovo anglicano John A.T. Robinson,
Honest to God, (trad. francese: Dieu sans Dieu, Paris, Nouvelles Éditions Latines, 1964; trad. italiana: Dio non è così, Firenze, Vallecchi, 1965), libro che diede inizio a violente polemiche. Se si pensa poi che l'editore francese della traduzione del libro di Robinson è, in campo cattolico, esponente dell'anticonciliarismo tradizionalista e dichiara, per bocca del prefatore Louis Salleron, di procedere alla pubblicazione per mettere in guardia i poveri lettori sprovveduti e vaccinarli dal veleno modernista (trad. franc. cit., pp. 8-9), si comprenderanno i fraintendimenti di fondo che possono sottostare ai richiami alla teologia della morte di Dio e si comprenderà oltremodo come la circolazione, diretta $\mathrm{o}$ mediata, di un autore quale Bonhoeffer agisca in modo inaspettato, almeno a livello di linguaggio ed immaginario, su di un pubblico ideologicamente molto diversificato.

(64) JA, p. 313, alla data 23 gennaio [1994]. 
È vero che Bauchau ritrovava questa nozione di faiblesse di Dio proprio nella riflessione sul versetto da lui prediletto della seconda lettera ai Corinzi:

«Ma grâce te suffit». Je me suis redit cela si souvent que j'avais oublié la suite dans l'Épître de saint Paul: «Ma puissance donne toute sa mesure dans la faiblesse». Ou, suivant la version de Chouraqui: «Oui, parfaite est la puissance dans la faiblesse». Ainsi la faiblesse que je ressens si fort est le lieu même où peut se manifester la puissance divine. Le mot puissance me surprend, je préférerais force. Mais dans puissance il y a c'est vrai, une majesté qui nous soulève et parfois nous transporte ${ }^{65}$.

Qui tuttavia, nell'appunto su Marguerite Yourcenar, potrebbe rivelarsi un influsso generico - o meglio una ricezione inconscia - di quella teologia della kénosis divina che è fondamento di tanta riflessione religiosa degli anni della secolarizzazione e del dopo-Auschwitz ${ }^{66}$, in un'insistenza tragica sull'impotenza e sulla debolezza di Dio. Basti pensare alla centralità del tema nei tentativi di ridefinizione del silenzio di Dio (dopo Nietzsche o Heidegger) negli scritti di prigionia di un Bonhoeffer che ripetutamente insiste su immagini kenotiche:

Les hommes vont à Dieu dans sa misère,

Le trouvent pauvre et méprisé, sans asile et sans pain,

Le voient abîmé sous le péché, la faiblesse et la mort.

Les chrétiens sont avec Dieu dans sa Passion ${ }^{67}$.

Devant Dieu et avec Dieu, nous vivons sans Dieu. Dieu se laisse déloger du monde et clouer sur la croix. Dieu est impuissant et faible dans le monde, et ainsi seulement il est avec nous et nous aide. Mat., 8, 17 indique clairement que le Christ ne nous aide pas par sa toutepuissance, mais par sa faiblesse et ses souffrances ${ }^{68}$.

D'altra parte, se vogliamo fare emergere intertestualità e commistioni lessicali e semantiche, vale forse la pena richiamare proprio quel Bonhoeffer che coglie del cristianesimo sia la dimensione ascensionale sia la presenza e l'incidenza sul mondo occidentale $^{69}$, e dal cui immaginario (o da un immaginario consimile e diffuso) Bauchau potrebbe derivare alcune significative consonanze. Bonhoeffer (almeno con tre opere: Widerstand und Ergebung: Résistance et soumission, Gemeinsames Leben: De la vie communautaire, Ethik: Éthique) aveva avuto diffusione straordinaria e fortuna, a partire appunto dagli anni sessanta: anche, a volte, eco scandalistica all'interno di quel cattolicesimo erede di quello in cui Bauchau si era formato, quel cattolicesimo - perlomeno istituzionale - superato peraltro nell'assunzione del discorso psicanalitico.

Del resto, proprio la consonanza di immaginario che si stabilisce a partire dal lessico, e che riattualizza non solo il discorso ermeneutico scritturale in genere ma

(65) JJ, p. 169, alla data 25 settembre [1986].

(66) Cfr. H. Jonas, Il concetto di Dio dopo Auschwitz. Una voce ebraica, Genova, Il Melangolo, 1990 (Der Gottesbegriff nach Auschwitz. Eine judische Stimme, Frankfurt am Main, Suhrkamp Verlag, 1987: trad. it. di C. Angelino).

(67) Per questo e per gli altri testi bonhoefferiani si cita dalla traduzione francese degli anni sessanta, cui Bauchau, con buona probabilità, può avere avuto accesso. Si tratta qui di una delle poesie della prigionia nel campo di Tegel (Chrétiens et païens), contenute in Résistance et soumission (Widerstand und Ergebung), trad. française de L. Jeanneret, Genève, Labor et Fides, 19672, p. 168.

(68) Ibid., p. 162.

(69) Cfr. D. BonHoeffer, Éthique, trad. française de L. Jeanneret, Genève, Labor et Fides, 1965, p. 92 : «De même que la responsabilité d'avoir abandonné le Christ est commune à l'Occident, aussi variable que puisse être la culpabilité d'un pays à l'autre, de même il n'y aura de justification et de renouvellement de l'Occident que communautaires. Toute tentative de sauver l'Occident à l'exclusion d'un des peuples occidentaux sera vouée à l'échec». 
in particolare quello costruito su alcune delle riflessioni teologiche di Bonhoeffer, è al riguardo particolarmente illuminante. Raffrontiamo, pertanto, ancora un passo di Bauchau con uno di Bonhoeffer:

[...] la dictée intérieure ${ }^{70}$ de la création naît de l'intense loisir du silence pour aller vers celui de l'écoute. Dans l'intervalle, il y a l'inspiration de la table de travail car le côté divin de la poésie est antérieur au poème qui se dirige non vers les dieux mais vers ce qu'il y a de plus humain dans l'homme ${ }^{71}$.

Le silence du chrétien est un silence plein d'attention, humble, et qui, à cause de son humilité, accepte d'être interrompu. Il est en liaison avec la parole. [...] Il y a dans le silence une puissance de clarification, de purification et de compréhension de l'essentiel. C'est déjà un fait sur le plan purement profane. Savoir se taire avant d'entendre la parole de Dieu, conduit à bien l'entendre et, par là même, à la dire à propos ${ }^{72}$.

Ora, come la creazione poetica nasce da un intimo dettato del profondo nutrito di silenzio, teso all'ascolto dell'universo interiore ${ }^{73}$, così il messaggio biblico può essere interiorizzato, vissuto pienamente e attualizzato a condizione che ci si metta in tacita relazione con la Parola che è accoglienza nell'ascolto. Pertanto, come in una prospettiva biblico-teologica la Parola diventa operante nella vita del cristiano, così il «côté divin de la poésie» rende possibile nel poeta l'innesto dei processi dell' «inspiration», ricuperando «ce qu'il y a de plus humain dans l'homme», cioè, probabilmente, la sua bellezza originaria, quella che, nonostante tutto, riflette bagliori della trascendenza divina. Le immagini letterarie coagulano nell'incalzare di un lessico simbolico e di una messe di intrecci linguistici:

[...] c'est un moment de bonheur où je communique avec une profondeur, avec un immense passé, tout en me dirigeant, de façon imprécise mais certaine, en avant. Ce bonheur, ce leurre offert à mon espoir par un amour véritable, mais qui doit demeurer ignoré, est nécessaire pour que je continue à poursuivre mon entreprise ou mon voyage. Car entretemps j'ai plus au moins perdu de vue mes perceptions initiales. L'esprit n'est plus orienté vers un but mais par le désir de s'enfoncer - et peut-être de se perdre - dans une matière. Matière verbale, matière d'images, de sons et de sens. Matière de l'écriture elle-même qui est toujours pour moi matière féminine. Cette matière attire l'esprit, le capte, le lie à l'œuvre, à la table de travail et à la né-

(70) Bauchau ritorna sull'idea del dettato interiore come momento che prende corpo «dans la partie intermédiaire du roman [...]. Ce stade élémentaire, continua Bauchau, est fondamental, c'est là que s'opère la dictée intérieure initiale» (cfr. $J A$, p. 358, alla data 1 ottobre [1994], le sottolineature sono nostre). Dettato interiore che è anche «dictée inconsciente» (JA, p. 405, alla data 28 maggio [1995]).

(71) EC, p. 16 (le sottolineature sono nostre).

(72) Cfr. D. BonhoefFer, Gemeinsames Leben, Chr. Raiser Verlag, München, 1939 (trad. fr. de F. Ryser: De la Vie communautaire, Genève, Delachaux et Niestlé, 1968, p. 79).

(73) Il tema dell'ascolto interiore come momento indispensabile alla creazione poetica è, come abbiamo ripetutamente detto, ricorrente. Bauchau infatti ne sottolinea la centralità: «[...] je me sens guidé par un rythme d'abord confus mais auquel je dois me conformer, par un son de voix que je reconnais peu à peu pour le mien lorsque j'ai la fermeté suffisante pour l'attendre et l'écouter» $(E C$, p. 14$) ; \ll[\ldots]$ il écoute [Jean Amrouche, un amico di Bauchau] et c'est bien ce que fait le poète, qui doit laisser monter en lui la forme venue de l'inconnu, et sans doute antérieure à la conscience, avant de pouvoir y inscrire sa parole» (Des Instruments spirituels, in EE, p. 76). Sempre in riferimento allo stesso tema Bauchau dichiara: «[...] je ne suis pas quelqu'un qui cherche et qui sait mais seulement quelqu'un qui écoute - un peu confusément d'ailleurs - et qui va» $(J A$, p. 124 , alla data 28 ottobre [1991]). Ascolto che passa in prima istanza attraverso la percezione fisica, quella che si realizza grazie all'orecchio, considerato filtro innocente, e per questo attraversato dalla purezza veicolata dalla poesia: «[...] c'est l'oreille d'abord qui perçoit. Pourquoi l'oreille? Parce que c'est elle qui, déjà dans le ventre maternel, entend le bruit, le rythme fondamental du cœur et les premières voix, celle de la mère et celle du père. C'est donc l'innocence de l'oreille qui peut entendre ce qui n'a pas encore été entendu et se prosterner plus profond» (L'Innocence de l'oreille, in EE, p. 155). Ma le citazioni potrebbero moltiplicarsi, poiché l'autore ritorna spesso su questo argomento sia in Jour après jour sia nel Journal d'Antigone. 
cessité d'un intense loisir qui le force à mettre douloureusement entre parenthèses ses autres préoccupations. La poésie dévaste la vie courante, la dénude et déborde le poète. Le poème souvent perd le souvenir de la source et la direction de l'estuaire. Il m'amène parfois à vivre, à comprendre, à dire tout autre chose que ce que je voulais exprimer en commençant. À perdre la vision première, initiante ou initiatique qui devait m'aider à me découvrir, à retrouver l'objet perdu et à inventer, au-delà de sa banalité, la vérité de l'existence ${ }^{74}$.

È però un coagularsi all'interno di un discorso che si fa reminiscenza di tutta una formazione spirituale cattolica e di un sostrato biblico-linguistico dalle forti connotazioni semantiche («amour véritable qui doit demeurer ignoré», «s'enfoncer, se perdre dans la matière», «la poésie dévaste la vie courante, la dénude et déborde le poète», «perdre le souvenir de la source et la direction de l'estuaire», «perdre la vision première», «retrouver l'objet perdu», ecc.), e in questo discorso Bauchau esprime quel barlume di felicità, pur effimero e ingannevole («ce leurre offert à mon espoir»), che si lega all'intensità di un momento («c'est un moment de bonheur où je communique...»). Bonbeur che sprigiona però non dalla ricerca isolata di un unico istante di creazione poetica, ma dalla quête incessante ( «je continue à poursuivre mon entreprise ou mon voyage») che travalica il singolo momento e che tende al ricupero della «vérité de l'existence» attraverso l'ispirazione.

Anche Bonhoeffer, in una delle sue poesie dal carcere, là dove pone l'interrogativo sulla felicità e sull'infelicità, ritrova nell'ora della 'comunicazione' («moment», per Bauchau, ov'egli «communique»; «l'heure [qui] sonne», per il teologo tedesco) il 'rischiararsi' di ogni infelicità:

Bonbeur, malheur, qu'est-ce donc?

[...].

Alors sonne l'beure de la fidélité,

[...]

Quel que soit le malheur,

La fidélité l'éclaire

Et légèrement l'enveloppe

D'un éclat doux et celeste (le sottolineature sono nostre $)^{75}$.

Condensato in una gamma ricchissima di echi scritturali, questo dinamico spostamento figurato, idealmente rappresentato da una linea immaginaria di unione che da uno spazio interiore diverge verso uno spazio esteriore, da uno stato in sé a uno fuori da sé, richiama la teologia, l'esegesi biblica e la riflessione omiletica cristiana. In questa prospettiva ci basti isolare due frammenti per identificare la portata dello spostamento figurato all'interno del discorso: «bonbeur, ce leurre offert à mon espoir par un amour véritable mais qui doit demeurer ignoré», e poi ancora «la poésie dévaste la vie courante, la dénude et déborde le poète». In particolare, il bonbeur - cui già accennavamo -, seppur illusorio in quanto dominato dal transeunte che permea la condizione umana, è radicato in un amore che non a caso è «amour véritable» connesso all'«espoir», quell'«espoir»/«espérance» che, altrove, Bauchau connette col «sens dans l'existence»:

Non sans bien des divagations, je cherchais partout l'espérance. Je l'ai cherchée surtout dans mon œuvre. L'espérance qui la soutient, particulièrement dans CEdipe sur la route, est

(74) EC, p. 15.

(75) Cfr. la poesia Bonbeur et malheur, contenuta in Résistance et soumission, trad. cit., p. 150. L'istantaneità del momento felice è ribadita in un altro testo poetico bonhoefferiano (le Stations sur le chemin de la liberté) che Bauchau avrebbe potuto leggere in quanto anch'esso accluso in Résistance et soumission, trad. cit., p. 171: «Un seul instant tu atteignis à la joie de la liberté» (anche se il testo in questione continuando «puis tu la remis à Dieu» orienta, diversamente che in Bauchau, la riflessione verso la trascendenza). 
fondée sur ma confiance, en lutte constante avec un fond de scepticisme, dans l'existence non pas d'un but, mais d'un sens ${ }^{76}$.

La poesia sconvolge («dévaste»), spoglia («dénude») e va oltre («déborde») i confini del poeta, come l'amore disarma, libera e travolge: è questa l'affermazione centrale del passo dell'Écriture à l'écoute testé citato. L'«amour véritable» di Bauchau è qui stilema scritturale, in quanto può echeggiare quel vero Amore che la Scrittura e la teologia identificano solo nell'amore di Dio, il cui volto «demeure ignoré», nonostante Egli si sia rivelato in Cristo suo figlio ${ }^{77}$.

La scelta lessicale poi di un morfema come «déborde» ${ }^{78}$ richiama esplicitamente l'idea dello straripamento, dunque una realtà referenziale che, in prospettiva semasiologica, attinge dall'immaginario veicolato dal significante acqua. Acqua il cui simbolismo, per di più, se ricondotto ai morfemi «initiante»/«initiatique», non può non ricuperare o farsi eco della simbologia dell'acqua salvifica, così come essa emerge dalle interstualità ricorrenti fra Antico e Nuovo Testamento. In particolare, se noi isoliamo in Bauchau la nozione di acqua salvifica, sentiamo l'eco scritturale di questo lessema ${ }^{79}$.

(76) JJ, p. 302, alla data 3 settembre [1989].

(77) Il tema del Deus absconditus, connotante una delle filières della teologia biblico-cristiana, è al centro della riflessione teologica dell'ultimo secolo. Per una sintesi chiarificatrice riportiamo qui un passo di Bruno Forte che ben sintetizza il discorso connesso a tale riflessione: $\ll[\ldots]$ il Dio dell'intera testimonianza biblica è un Dio in esodo da sé, un Dio che ha avuto tempo per l'uomo $\mathrm{e}$, venendo nella storia, ha stretto alleanza con lui, dischiudendo il cammino del suo popolo verso il Regno promesso, sempre più grande di qualunque compimento realizzato. Ė il Dio che, pur comunicandosi nelle parole e negli eventi della storia della salvezza, resta sempre al di là di ogni presa umana. Perciò, il Suo avvento è "ri-velazione": uno svelarsi che vela, un venire che apre il cammino, un ostendersi nel ritirarsi che attira. Questa dialettica di apertura e di nascondimento raggiunge il suo vertice nell'autocomunicazione personale di Dio nel Figlio incarnato: la Parola che si dice nelle parole rinvia all'abissale Silenzio da cui viene. Il Dio in carne umane è al tempo stesso rivelato e nascosto, revelatus in absconditate et absconditus in revelatione [...]. La rivelazione del Dio che viene toglie il velo che cela, ma è anche un più forte nascondere, è comunicazione di sé, che inseparabilmente si offre come nuovamente "velare". Si può affermare, allora, che la tradizione ebraico-cristiana comprende - accanto a una teologia della Parola, inseparabile da essa - una teologia del Silenzio: il dire di Dio non si compie mai senza un Suo più alto tacere. Il silenzio non è solo il grembo fecondo dell'avvento, ma anche il luogo del compimento finale, quando "Dio sarà tutto in tutti" e ogni creatura sarà finalmente e pienamente se stessa in Lui. Verso questa Patria anela il silenzio dell'attesa: il Dio silenzioso e raccolto è la vocazione del mondo, l'approdo della nostalgia inscritta nell'essere silente della creatura. Dal Silenzio al Silenzio: in questa formula potrebbe evocarsi il disegno divino, che unisce l'inizio e il compimento degli esseri, tesi a Colui da cui provengono. Il mistero di Dio è la custodia silenziosa in cui tutto vive sin dal primo mattino del mondo, il grembo da cui veniamo, in cui ci muoviamo e siamo, il Volto e l'abbraccio verso cui andiamo. Sullo sfondo di questo Silenzio divino il Verbo che viene nella carne si offre come la luce nelle tenebre, la rivelazione dell'amore eterno attua nella "consegna" di sé sino alla fine, il Figlio che ci rende figli aprendoci l'accesso al mistero del Padre, Silenzio dell'origine e della patria finale» (cfr. B. FORTE, L'essenza del Cristianesimo, Milano, Mondadori, pp. 43-44).

(78) L'immagine dell'acqua ritorna nel lessico bauchauliano ancora attraverso l'uso del morfema déborder - ma anche di altri lessemi isolati o ancora di sintagmi più complessi -, questa volta però in riferimento all'ispirazione poetica: «[...] la Poésie doit soulever l'obstacle intérieur et extérieur, elle doit poursuivre son cours en le franchissant ou plus exactement en le débordant. Pour les moments où le flot passe et déborde dans l'inspiration, il faut les longues heures de préparation, de travail obscur, affronter les turbulances des courants et les risques d'inondation» (JA, p. 254, alla data 21 giugno [1993]. Le sottolineature sono nostre). Sempre per l'uso della metafora acquatica in relazione ai fenomeni creativi tipici della scrittura cfr. $I A$, p. 17 , alla data 25 novembre [1989]: «il s'agit alors de laisser monter en soi ce qui vient d'une source inconnue $[\ldots .] »,. J A$, p. 441, alla data 4 gennaio [1996]: «c'est l'état de santé de L. qui, l'année dernière, m'a orienté pendant plusieurs mois vers la poésie, pour me ressourcer et reprendre courage. Le courage est en effet le thème essentiel de cette vague d'inspiration poétique» (le sottolineature sono nostre). L'immagine dell'acqua è sfruttata poi come immagine ispiratrice, per la costruzione di poemi più o meno complessi o di semplici riflessioni pressoché estemporanee: cfr. $P B G$, pp. $40-42$, alla data 14 gennaio [1998]; pp. 48-49, alla data 9 febbraio, [1998]; p. 50 , alla data 21 febbraio [1998]; p. 84, alla data 29 settembre [1998]; p. 92, alla data 18 dicembre [1998]; p. 329, alla data 17 marzo [2001].

(79) Innumerevoli sono i riferimenti scritturali 
Ora, è vero che le consonanze lessicali, e di immaginario da noi evidenziate potrebbero essere estendibili a scritti teologici di diversa origine (abbiamo qui evocato Bonhoeffer per il suo essere, negli anni sessanta, segno di contraddizione, all'interno di un vasto dibattito teologico e 'politico' a un tempo). Le riflessioni che sono state isolate sono anche al centro di un'esegesi biblica e di una riflessione teologica secolare, e pur ipotizzando un approccio diretto al pensiero di teologi contemporanei da parte di Bauchau, resta evidente che semplici echi intertestuali non possono costituire prova di una formazione sicuramente definibile dell'autore belga su precisi testi della spiritualità novecentesca. È altrettanto vero, però, che, al di là delle specifiche letture, i rinvii e i parallelismi semantico-ideologici, che appartengono talvolta a un lessico biblico e teologico, sottostanno al processo di elaborazione poetica bauchauliano in un fitto gioco retorico di reminiscenze appunto, costruite su tre momenti centrali che fanno capo a un profondo e complesso dinamismo ciclico-compositivo: 1) analisi/ scrittura; 2) incoscio/conscio; 3) vita/analisi, con un richiamo non meno importante a quelle implicazioni esistenziali nella scrittura che si caricano di connotazioni personali, proprio nel riferimento, forse consapevole forse involontario, alla teologia e alle Scritture. Pertanto i due livelli semantici, cui si faceva cenno in apertura, sono evidenziati - è questo l'assunto del presente lavoro - dal continuo glissement de sens che contraddistingue quell'intrecciarsi di intertestualità caratterizzanti la profonda concettualità di un'opera che si pone anzitutto come letteraria ${ }^{80}$.

Nota CRONOLOGICO-BibliografiCA. Abbiamo tentato, in parte, di ripercorrere l'elaborazione di una poetica. Tuttavia, Bauchau non è autore che costruisca in modo sistematico la sua riflessione teorica. Narratore, poeta e moralista, egli affida questa riflessione all'annotazione e al frammento occasionale, spesso senza seguire un piano organico tale da permettere di ricostruire un'evoluzione di pensiero che si snodi per tappe, definitive nel segnare un filo ininterrotto di teoresi; ma offrendo piuttosto, attraverso un continuo ripiegarsi diaristico sul proprio laboratorio, testimonianze e considerazioni concernenti un'officina letteraria in fieri. Per questo la nostra lettura muove dal frammento al frammento, a volte senza un ordine che non sia quello della tematica o della rispondenza intertestuale. Conviene, pertanto, avere ben chiara la successione dei testi cui si fa riferimento, e pertanto si ritiene opportuno offrire una cronologia dei titoli bauchauliani qui sfruttati:

La Grande Muraille. Journal de la «Déchirure» (1960-1965): la stesura del testo, pubblicato nel 2005, risale agli anni 1960-1965 (GM).

La Sourde Oreille ou le rêve de Freud: 1981 (SO).

Des Instruments spirituels: prefazione di un libro di Jean Amrouche del 1986, pubblicata in EE.

Rencontres avec Freud: pubblicate in EE, comparvero nelle «Études freudiennes» nel 1986.

L'Écriture et la Circonstance: contiene una serie di quattro conferenze tenute nell'ottobre 1987 (EC).

Jour après jour. Journal d'«EEdipe sur la route» (1983-1989): la stesura del testo, pubblicato nel 1992, risale evidentemente agli anni coperti dal diario $(J J)$.

Journal d'«Antigone» (1989-1997): la stesura del testo, pubblicato nel 1999, risale evidentemente agli anni coperti dal diario $(J A)$.

L'Innocence de l'oreille: contributo pubblicato in EE, compare per la prima volta nel 1999.

Passage de la Bonne-Graine. Journal (1997-2001): la stesura del testo, pubblicato nel 2002, risale evidentemente agli anni coperti dal diario $(P B G)$.

$L a$ Scène du rêve: conferenza pubblicata in EE, letta in un convegno delle «Études freudiennes» nel gennaio 2000 .

MICHELE MASTROIANNI

che si potrebbero addurre. Citiamo solo Joan., 4, 7-16; 7, 38; Apoc., 7, 17; 21, 6; 22, 1; 22, 17.

(80) Questo lavoro deve molto all'assiduo con- fronto con Chiara Maurella, che ha accettato di rileggere attentamente le mie pagine. 